\title{
Simulation of the droplet-to-bubble transition in a two-fluid system
}

\author{
M. Garzon, ${ }^{1, *}$ L. J. Gray, ${ }^{2}$ and J. A. Sethian ${ }^{3}$ \\ ${ }^{1}$ Department of Applied Mathematics, University of Oviedo, Asturias, Spain \\ ${ }^{2}$ Computer Science and Mathematics Division, Oak Ridge National Laboratory, Oak Ridge, Tennessee, USA \\ ${ }^{3}$ Department of Mathematics, University of California, Berkeley, California, USA and Mathematics Department, \\ Lawrence Berkeley National Laboratory, Berkeley, California, USA
}

(Received 24 December 2009; revised manuscript received 2 March 2011; published 27 April 2011)

\begin{abstract}
Recent experiments by Burton and Taborek have demonstrated a droplet-to-bubble transition in the pinchoff behavior of one inviscid fluid inside another. With $D$ the relative densities $\rho_{\mathcal{E}} / \rho_{\mathcal{I}}$, they find transition from ( $D=0$ ) droplet-to-bubble behavior at $D \approx 4$. Numerical simulations of this two-fluid system, up to and beyond the initial breakup of the inner fluid, have been carried out utilizing level set and boundary integral methods. A droplet-to-bubble transition is predicted: For $D$ sufficiently large, the volume of the satellite droplet shrinks to zero and there is no overturning of the fluid at separation. The calculated self-similar scaling exponents and the pinchoff region shapes match the known behavior at the droplet and bubble extremes $(D=0, D=100)$. For intermediate $D$ values, the simulations presented here indicate that the transition range between droplet and bubble behavior depends upon initial drop geometry. When the neck separates two nonequal inner fluid masses the transition is mild and occurs in the range $4<D<10$, whereas in the case of equal masses an abrupt transition occurs at $D \approx 4$ in perfect agreement with the above mentioned experiments.
\end{abstract}

DOI: 10.1103/PhysRevE.83.046318

PACS number(s): 47.55.db, 47.15.km, 47.11.Hj, 47.11.Bc

\section{INTRODUCTION}

In part due to many important technological applications [1], fluid breakup has been extensively studied by means of experimental [2], theoretical, and computational analysis [3,4]. Recently, Burton and Taborek [5] have employed a high speed camera to study the pinchoff of xenon immersed in water. By varying the density of the xenon, they were able to examine the pattern of fluid pinchoff and the scaling exponents as a function of the ratio of the fluid densities, $D=\rho_{\mathcal{E}} / \rho_{\mathcal{I}}$, where $\mathcal{E}$ and $\mathcal{I}$ indicate the exterior (water) and interior (xenon) fluids. They found that there appeared to be a sharp transition in the pinchoff behavior: For $D<4$ the scaling exponents are as in the $D=0$ Rayleigh limit and satellite droplets immediately appear, while for $D>4$ the exponents differ and only a single "bubblelike" separation occurs. Using a potential flow model, the present work studies the two inviscid fluid evolution through pinchoff into satellite breakoff and the development of substructures, for a wide range of $D$ values. For low viscosity fluids the potential flow assumption is valid up to the nanometers length scale. Previous numerical approximations of the same model equations [6,7], based on marker particles, do not present results after pinchoff, which is probably due to the inherent difficulties of these methods to follow free boundary topological changes. Moreover, for $D>6$ numerical instabilities reported in [6,7] are absent in the present calculations.

In a recent work [8], the collapse of a single inviscid fluid column ( $D=0$, Rayleigh instability of a liquid jet) has been numerically approximated. The algorithm combines a level set method [9] for advancing the free surface and the free surface boundary condition, together with a boundary integral approach for the evaluation of surface velocities. This approach was successful in modeling the free surface

*maria@ orion.ciencias.uniovi.es evolution, continuing the simulation beyond initial separation and through the subsequent satellite drop evolution, while accurately predicting the known scaling exponent, $\alpha_{D}=2 / 3$, of the self-similar power law $r \propto \tau^{\alpha_{D}}$. Here $r$ is the minimum neck radius and $\tau$ is the time remaining until pinchoff.

The model and numerical approximation introduced in the current work allow us to consider the entire range of values for $D$, including the $D=0$ case. In particular, see the following:

(1) We introduce a methodology to pass through breakup and compute dynamic motion after topological change. Mechanism of satellite formation, evolution, and satellite size can be analyzed.

(2) The resulting algorithm provides a seamlessly unified method for computing across a wide range of $D$ values and no numerical instabilities were present for $0 \leqslant D \leqslant 100$.

(3) In the case $D=0$, the two-fluid solution matches the results in [8] and produces the known behavior at pinchoff including correct scaling exponents and overturning of the fluid.

(4) We are able to compute scaling exponents over the entire range of $D$ values. The similarity exponents and computed profiles are consistent with those predicted by theories and reported in recent experiments.

The initial drop shape, which was taken as in [6] for comparison, is initially at rest with a neck already formed separating two nonsymmetrical fluid masses. For the values of $D$ considered herein for this case, the transition from satellite-to-bubble behavior (no satellite) occurs in the range $4<D<10$. This wider range, which differs from the sharper range $(D \approx 4)$ found in [5] made us conjecture that it could be due to differences between their experimental settings and our model physical assumptions or model initial drop geometry. To further investigate this fact we run a new set of simulations starting with an initial drop whose initial neck separates two equal fluid masses. For this drop geometry a sharp transition is found at $D \approx 4$, in perfect agreement with Burton and Taborek 


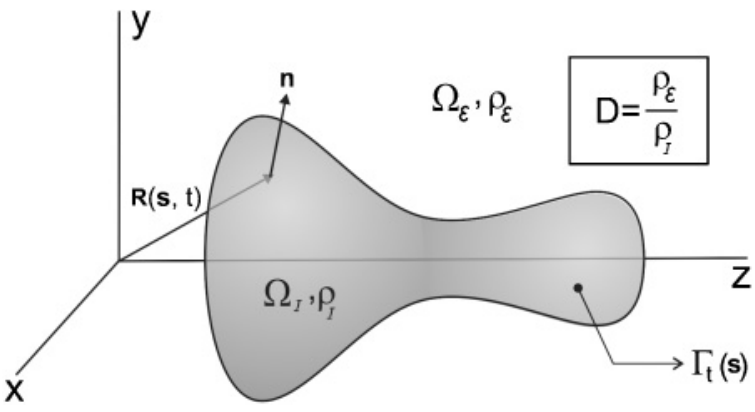

FIG. 1. Physical setting in three dimensions.

laboratory experiments. These two initial drop geometries are depicted in Figs. 2 and 3 and will be referred to as geometry 1 and 2 , respectively.

\section{MATHEMATICAL MODEL}

The presentation below is kept as brief as possible; further details can be found in $[8,10,11]$. Consider a fluid of density $\rho_{\mathcal{I}}$ immersed in an (infinite) exterior fluid of density $\rho_{\mathcal{E}}$. The system is initially at rest and, in absence of gravity, the fluid movement is induced by surface tension forces.

Let $\Omega_{k}(t), k=\mathcal{I}, \mathcal{E}$, be the three-dimensional (3D) interior and exterior fluid domains, respectively, $\Gamma_{t}(\mathbf{s})=$ $(x(\mathbf{s}, t), y(\mathbf{s}, t), z(\mathbf{s}, t))$ a parametrization of the free surface boundary at time $t$ and $\mathbf{R}(\mathbf{s}, t)$ the position vector of a fluid particle on the moving front (see Fig. 1). Assuming potential flow, the fluid velocities $\mathbf{u}_{k}$ for each fluid domain $\Omega_{k}(t)$, $k=\mathcal{I}, \mathcal{E}$ are given in terms of a potential $\phi_{k}$,

$$
\begin{gathered}
\mathbf{u}_{k}=\nabla \phi_{k}, \\
\Delta \phi_{k}=0, \\
D_{t} \mathbf{R}=\mathbf{u}_{\mathcal{I}} \text { on } \Gamma_{t}(\mathbf{s}) .
\end{gathered}
$$

The last equation is the kinematic boundary condition (for the interior fluid), which states that the interface moves with the fluid velocity, with $D_{t}$ denoting the total derivative following the fluid (interior) particles,

$$
D_{t}=\frac{\partial}{\partial t}+\mathbf{u}_{\mathcal{I}} \cdot \nabla
$$

On the free boundary between the two fluid domains, $\Gamma_{t}(\mathbf{s})$, the continuity of the normal velocity and normal stress tensor gives

$$
\begin{gathered}
\mathbf{n} \cdot \nabla \phi_{\mathcal{I}}=\mathbf{n} \cdot \nabla \phi_{\mathcal{E}} \\
\rho_{\mathcal{I}}\left(\frac{\partial \phi_{\mathcal{I}}}{\partial t}+\frac{1}{2}\left|\nabla \phi_{\mathcal{I}}\right|^{2}\right)-\rho_{\mathcal{E}}\left(\frac{\partial \phi_{\mathcal{E}}}{\partial t}+\frac{1}{2}\left|\nabla \phi_{\mathcal{E}}\right|^{2}\right)+\gamma \kappa=0
\end{gathered}
$$

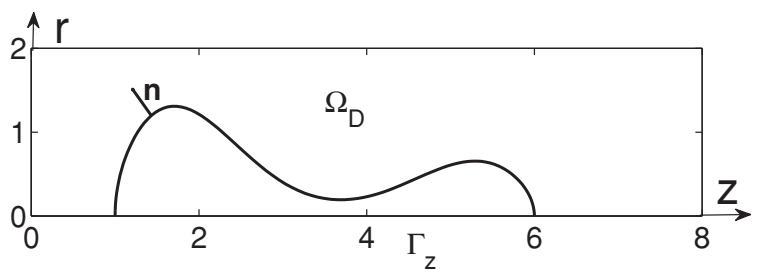

FIG. 2. Computational domain in the $r-z$ plane, drop geometry 1 .

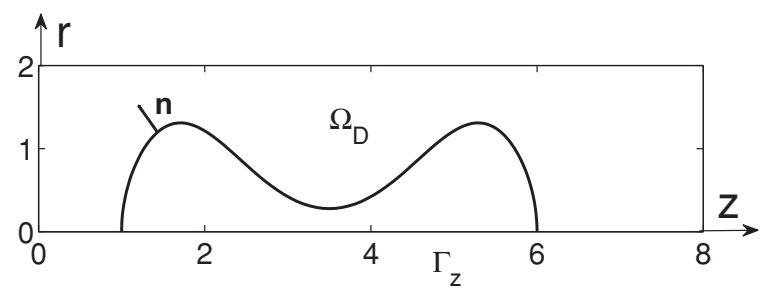

FIG. 3. Computational domain in the $r-z$ plane, drop geometry 2 .

where $\mathbf{n}$ is the unit normal vector pointing from the interior to the exterior domain, $\kappa=1 / R_{1}+1 / R_{2}$ is twice the mean curvature of the surface, $R_{1}, R_{2}$ is the principal radii of curvature, and $\gamma$ is the surface tension coefficient. Taking as characteristic length scale the initial neck radius $r_{0}$ and time scale $t_{0}=\left(\rho_{\mathcal{I}} r_{0}^{3} / \gamma\right)^{1 / 2}$ we get

$$
\left(\frac{\partial \phi_{\mathcal{I}}}{\partial t}+\frac{1}{2}\left|\nabla \phi_{\mathcal{I}}\right|^{2}\right)-D\left(\frac{\partial \phi_{\mathcal{E}}}{\partial t}+\frac{1}{2}\left|\nabla \phi_{\mathcal{E}}\right|^{2}\right)+\kappa=0,
$$

where now all the quantities are nondimensional. Next adding and subtracting the needed terms we obtain

$$
\begin{aligned}
& \frac{\partial \phi_{\mathcal{I}}}{\partial t}-D \frac{\partial \phi_{\mathcal{E}}}{\partial t}+\mathbf{u}_{\mathcal{I}} \cdot\left(\nabla \phi_{\mathcal{I}}-D \nabla \phi_{\mathcal{E}}\right) \\
& =\mathbf{u}_{\mathcal{I}} \cdot\left(\frac{1}{2} \nabla \phi_{\mathcal{I}}-D \nabla \phi_{\mathcal{E}}\right)+\frac{D}{2} \mathbf{u}_{\mathcal{E}} \cdot \nabla \phi_{\mathcal{E}}-\kappa
\end{aligned}
$$

and setting $\phi_{D}=\phi_{\mathcal{I}}-D \phi_{\mathcal{E}}$, we have

$$
\frac{\partial \phi_{D}}{\partial t}+\mathbf{u}_{\mathcal{I}} \cdot \nabla \phi_{D}=f \text { on } \Gamma_{t}(\mathbf{s})
$$

where

$$
f=\mathbf{u}_{\mathcal{I}} \cdot\left(\frac{1}{2} \mathbf{u}_{\mathcal{I}}-D \mathbf{u}_{\mathcal{E}}\right)+\frac{D}{2} \mathbf{u}_{\mathcal{E}} \cdot \mathbf{u}_{\mathcal{E}}-\kappa .
$$

The model equations in three dimensions $(k=\mathcal{I}, \mathcal{E})$ are therefore

$$
\begin{gathered}
\mathbf{u}_{k}=\nabla \phi_{k} \text { in } \Omega_{k}(t), \\
\Delta \phi_{k}=0 \text { in } \Omega_{k}(t),
\end{gathered}
$$

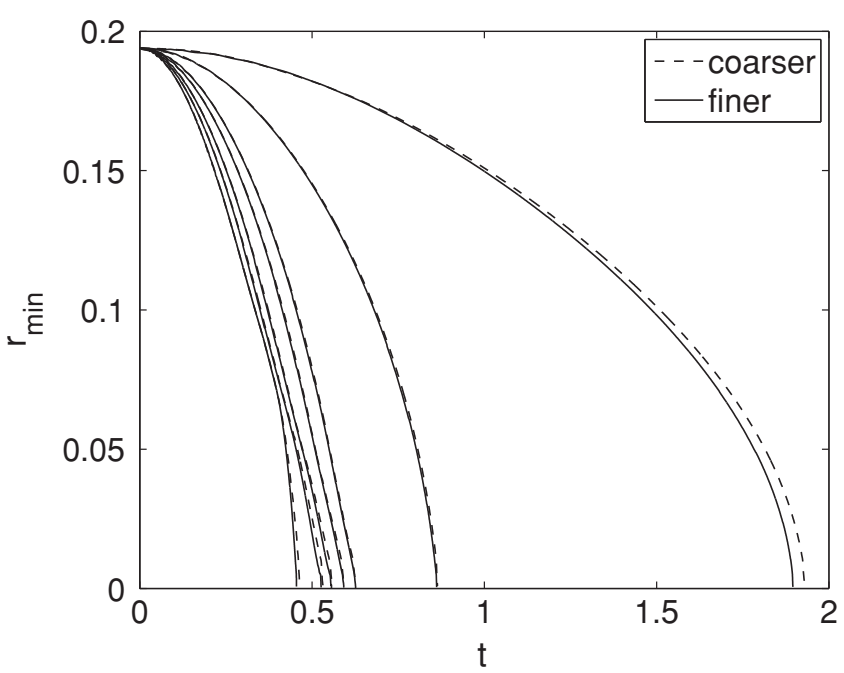

FIG. 4. Minimum neck radius time evolution for $D=$ $0,1,2,4,6,20,100$ (left to right). 
TABLE I. Pinchoff time and relative error in volume, geometry 1.

\begin{tabular}{lcccc}
\hline \hline$D$ & $t_{p}($ coarse $)$ & $t_{p}($ fine $)$ & $e_{V}($ coarse $)$ & $e_{V}($ fine $)$ \\
\hline 0 & 0.4551 & 0.4551 & $1.2036 \times 10^{-3}$ & $1.4499 \times 10^{-3}$ \\
1 & 0.5320 & 0.5263 & $1.0443 \times 10^{-3}$ & $5.5482 \times 10^{-4}$ \\
2 & 0.5585 & 0.5566 & $8.3183 \times 10^{-4}$ & $4.6596 \times 10^{-4}$ \\
4 & 0.5930 & 0.5923 & $5.9535 \times 10^{-4}$ & $4.6892 \times 10^{-4}$ \\
6 & 0.6265 & 0.6265 & $4.9328 \times 10^{-4}$ & $4.1447 \times 10^{-4}$ \\
20 & 0.8640 & 0.8620 & $4.4317 \times 10^{-4}$ & $2.2308 \times 10^{-4}$ \\
100 & 1.9300 & 1.8956 & $5.6643 \times 10^{-4}$ & $1.8306 \times 10^{-4}$ \\
\hline \hline
\end{tabular}

$$
\begin{aligned}
& D_{t} \mathbf{R}=\mathbf{u}_{\mathcal{I}} \text { on } \Gamma_{t}(\mathbf{s}), \\
& D_{t} \phi_{D}=f \text { on } \Gamma_{t}(\mathbf{s}) .
\end{aligned}
$$

This Lagrangian-Eulerian formulation is frequently approximated numerically using the so-called "front tracking method," which suffers difficulties when the free boundary changes topology. These problems are avoided using the level set formulation for both the kinematic and dynamic boundary conditions (7) and (8).

The remaining boundary condition needed to simultaneously solve the two Laplace equations, Eq. (6), is that the normal velocities in the two fluids are equal and opposite in sign. The far field condition for the exterior potential is set to zero.

\section{LEVEL SET EMBEDDING}

The level set method is a mathematical tool developed by Osher and Sethian [12] to follow interfaces which move with a given velocity field. The key idea is to view the moving front as the zero level set of a higher dimensional function, called the level set function. One main advantage of this approach comes when the moving boundary changes topology, and thus a simply connected domain splits into separated disconnected domains. Let $\Omega_{D}$ be a fixed $3 \mathrm{D}$ rectangular domain that contains the free boundary at any time $t$ and $\Gamma_{t}(\mathbf{s})$ the set of

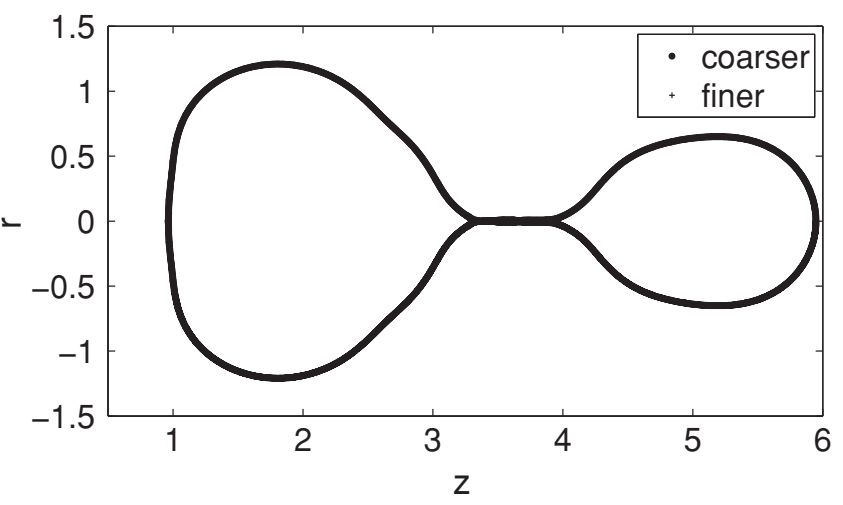

FIG. 6. Front profiles for $D=6$, coarser and finer grids.

points lying in the surface boundary at time $t$. This surface is defined through the zero level set of the scalar field $\Psi(x, y, z, t)$. An equation of motion for $\Psi$ that ties the zero level set of $\Psi$ to the evolving front comes from observing that the level set value of a particle on the front with path $\mathbf{R}(\mathbf{s}, t)$ must always be zero:

$$
\Psi(\mathbf{R}(\mathbf{s}, t), t)=0 .
$$

To embed the free surface boundary condition given by Eq. (8) into the level set framework, we define $G(x, y, z, t)$ on $\Omega_{D}$ such that

$$
G(\mathbf{R}(\mathbf{s}, t), t)=\left.\phi_{D}(x, y, z, t)\right|_{\Gamma_{t}(\mathbf{s})}=\phi_{D}(\mathbf{R}(\mathbf{s}, t), t) .
$$

Differentiating Eqs. (9) and (10) with respect to time, following the interior fluid particle characteristics, we have

$$
\begin{gathered}
\Psi_{t}+\mathbf{u}_{\mathcal{I}} \cdot \nabla \Psi=0, \\
G_{t}+\mathbf{u}_{\mathcal{I}} \cdot \nabla G=D_{t} \phi_{D}=f,
\end{gathered}
$$

which holds on $\Gamma_{t}(\mathbf{s})$. Note that $G(x, y, z, t)$ is an auxiliary function that can be chosen arbitrarily, the only restriction being that it is equal to $\phi_{D}(x, y, z, t)$ on the free surface. The
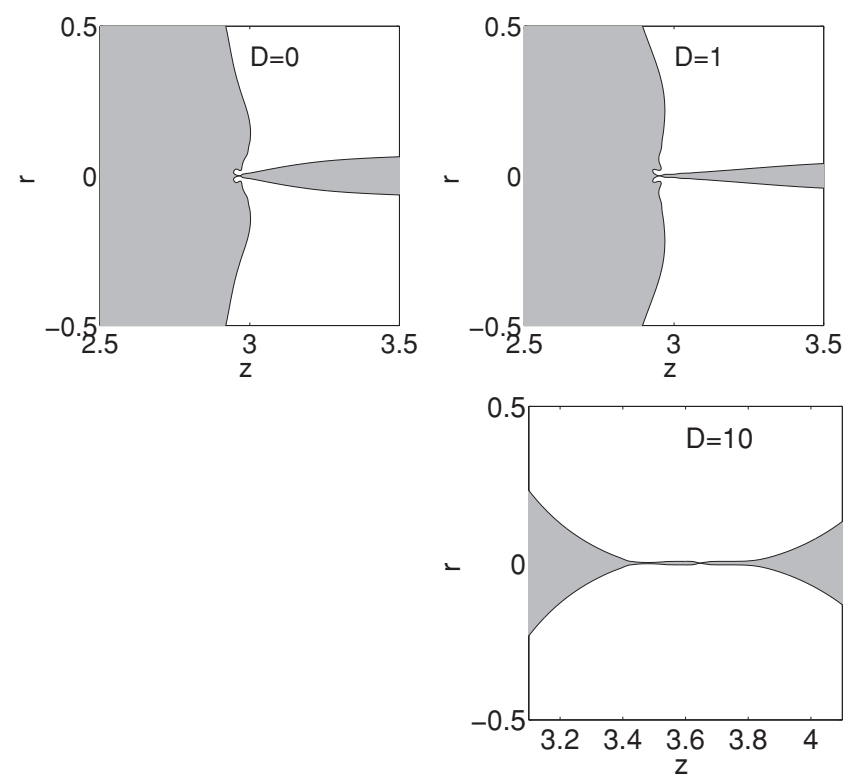
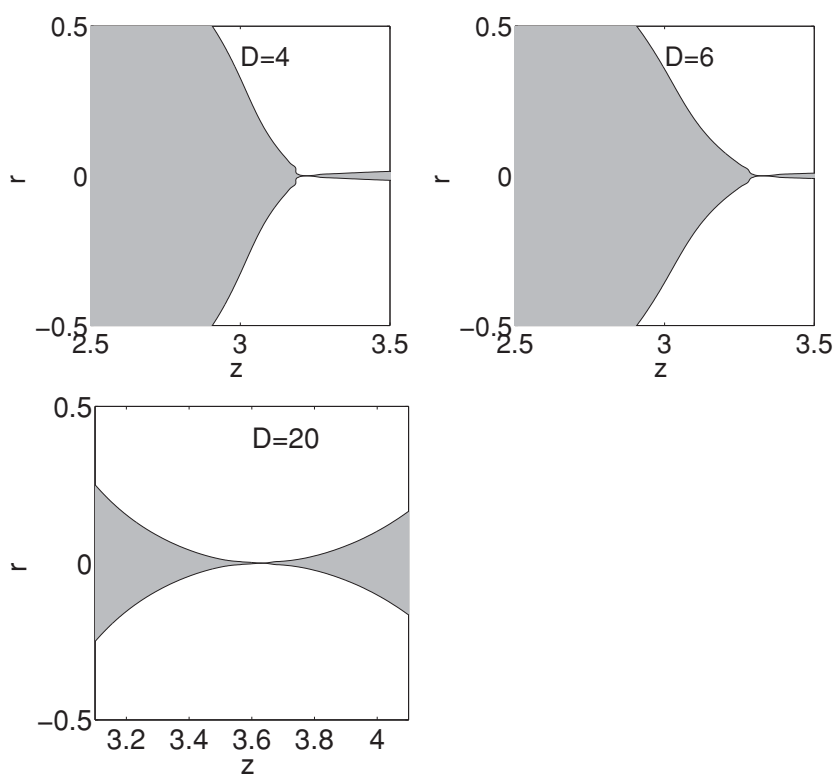

FIG. 5. Focused fronts at pinchoff. 


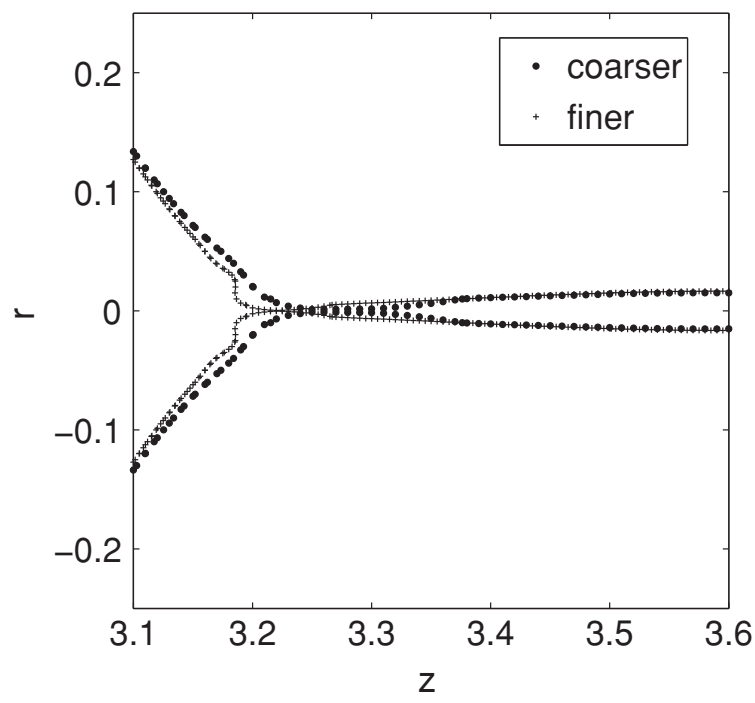

FIG. 7. Focused front profiles for $D=4$, coarser and finer grids.

velocity $\mathbf{u}_{\mathcal{I}}$ and the right-hand side of Eq. (12) are only defined on $\Gamma_{t}(\mathbf{s})$, and thus, in order to solve Eqs. (11) and (12) over the domain $\Omega_{D}$, these variables must be extended off the front. A detailed description of how to perform these extensions is given in [13]. The system of equations, written in a complete Eulerian framework $(k=\mathcal{I}, \mathcal{E})$, is

$$
\begin{gathered}
\mathbf{u}_{k}=\nabla \phi_{k} \quad \text { in } \Omega_{k}(t), \\
\Delta \phi_{k}=0 \quad \text { in } \Omega_{k}(t), \\
\Psi_{t}+\mathbf{u}_{\mathcal{I}_{\mathrm{ext}}} \cdot \nabla \Psi=0 \quad \text { in } \Omega_{D}, \\
G_{t}+\mathbf{u}_{\mathcal{I}_{\mathrm{ext}}} \cdot \nabla G=f_{\mathrm{ext}} \quad \text { in } \Omega_{D} .
\end{gathered}
$$

The subscript "ext" denotes the extension of $f$ and $\mathbf{u}_{\mathcal{I}}$ onto $\Omega_{D}$.

The free surface equations (7) and (8) have now been embedded into the higher dimension equations (15) and (16) and it can be shown that system (13)-(16) is equivalent to system (5)-(8). In fact, this enriches the kinematics of the system, in the sense that it can incorporate topological changes

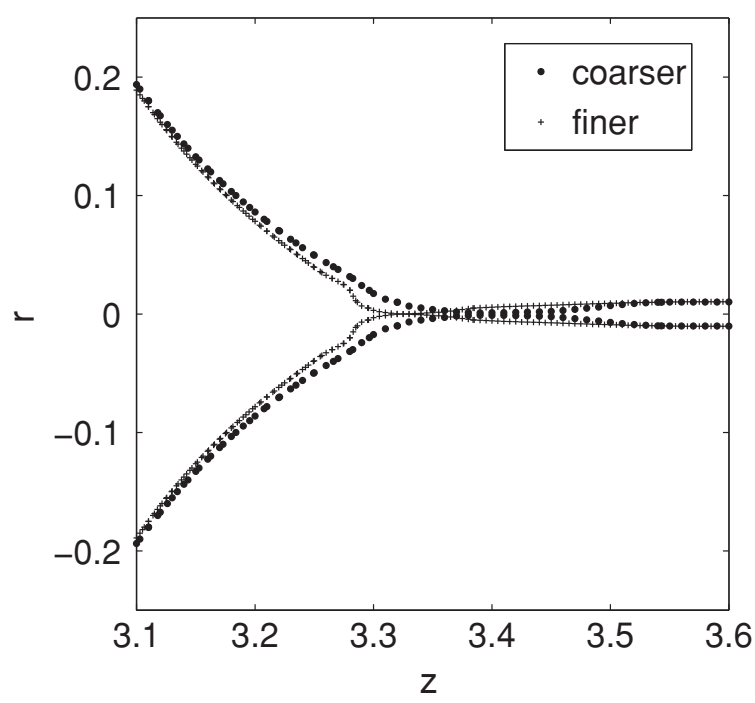

FIG. 8. Focused front profiles for $D=6$, coarser and finer grids.

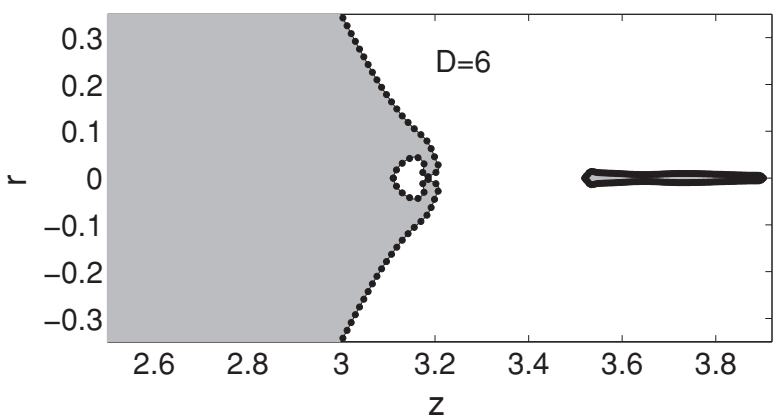

FIG. 9. Front focused at $t=0.635$ for $D=6$ showing BEM nodes.

of the free surface, and as well the evolution of the associated potential function within this boundary; see $[8,14]$.

Assuming symmetry around the $z$ axis the previous system can be formulated in two dimensions by writing the equations in cylindrical coordinates. The equations in the $(r, z)$ plane remain the same except the Laplacian that should be changed accordingly. In what follows axisymmetry will always hold and $\Omega_{k}(t), k=\mathcal{I}, \mathcal{E}$, will denote the $2 \mathrm{D}$ fluid domains in the $(r, z)$ plane, $\Gamma_{t}(s)$ the free boundary between these fluid domains, and $\Omega_{D}$ is a $2 \mathrm{D}$ fixed domain that contains the free boundary for all times.

\section{NUMERICAL APPROXIMATION}

The numerical approximation of system (13)-(16) in the $(r, z)$ plane can be described in two basic steps. First, using a standard first-order backward Euler explicit scheme to approximate time derivatives in the level set equations, the system to be solved for each time $t_{n}$ and time step $\Delta t, k=\mathcal{I}, \mathcal{E}$ is

$$
\begin{gathered}
\mathbf{u}_{k}^{n}=\nabla \phi_{k}^{n} \text { in } \Omega_{k}\left(t_{n}\right), \\
\Delta \phi_{k}^{n}(r, z)=0 \text { in } \Omega_{k}\left(t_{n}\right), \\
\frac{\Psi^{n+1}-\Psi^{n}}{\Delta t}=-\mathbf{u}_{\mathcal{I}_{\mathrm{ext}}} \cdot \nabla \Psi^{n} \text { in } \Omega_{D}, \\
\frac{G^{n+1}-G^{n}}{\Delta t}=-\mathbf{u}_{\mathcal{I}_{\mathrm{ext}}} \cdot \nabla G^{n}+f_{\mathrm{ext}}^{n} \text { in } \Omega_{D} .
\end{gathered}
$$

The second main task is to solve Eqs. (18) for the free surface velocity, subject to the boundary condition $\phi_{\mathcal{I}}^{n}-$ $D \phi_{\mathcal{E}}^{n}=G^{n}$. This is accomplished by solving the boundary integral equation corresponding to the Laplace equations in

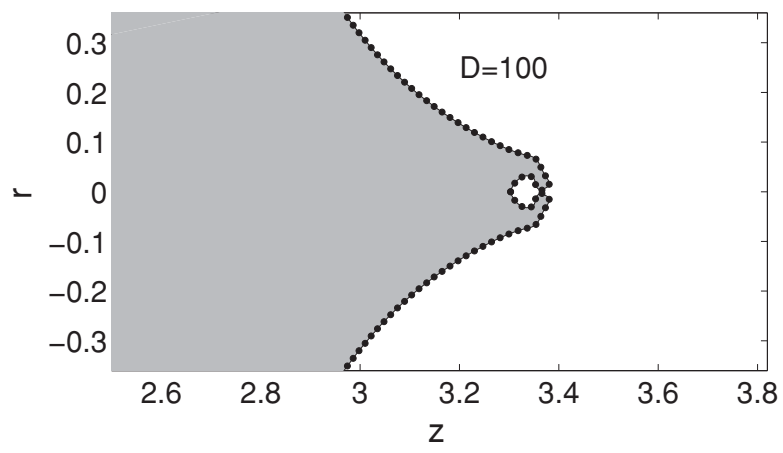

FIG. 10. Front focused at $t=1.89714$ for $D=100$ showing BEM nodes. 
TABLE II. Second pinchoff time, final time, relative error in volume, and total number of time steps, geometry 1 .

\begin{tabular}{lcccc}
\hline \hline$D$ & $t_{s}$ & $t_{f}$ & $e_{V}$ & $N_{\text {steps }}$ \\
\hline 0 & 0.46930 & 0.61430 & $2.4440 \times 10^{-3}$ & 2001 \\
0.3 & 0.48440 & 0.58445 & $1.0490 \times 10^{-3}$ & 2001 \\
1 & 0.55105 & 0.60635 & $1.3435 \times 10^{-3}$ & 1601 \\
2 & 0.57480 & 0.60675 & $1.1421 \times 10^{-3}$ & 1001 \\
4 & 0.60355 & 0.61095 & $1.0013 \times 10^{-3}$ & 360 \\
6 & 0.63325 & 0.63630 & $8.1248 \times 10^{-4}$ & 196 \\
20 & & 0.86420 & $3.4854 \times 10^{-4}$ & 121 \\
100 & & 1.89714 & $4.0577 \times 10^{-4}$ & 145 \\
\hline \hline
\end{tabular}

the axisymmetric geometry depicted in Fig. 2, the details to be discussed in Sec. IV C. With the computed velocity, the new position of the boundary is determined from the level set equation (19), and the potential $\phi_{D}$ on $\Gamma_{t_{n+1}}(s)$ will be obtained from Eq. (20). These procedures are described below.

\section{A. Initialization}

To initialize the system (17)-(20) the initial front position $\Gamma_{0}(s)=(r(s), z(s))$ and velocity potential $\phi_{D}(r, z, 0)$ are needed. For the initial front position we take the same geometry as in $[6]:^{1}$

$$
\begin{gathered}
z(s)=1+B[1-a(s)], \\
r(s)=g_{1}(s)+g_{2}(s), \\
a(s)=[\cos (s)+1] / 2,
\end{gathered}
$$

and for $0<s<\pi$ we set

$$
\begin{gathered}
g_{1}(s)=\sqrt{B} \sqrt{a(s)}\left(e^{-B^{2}[a(s)]^{2} / 2}-e^{-B^{2} / 2}\right), \\
g_{2}(s)=C \sqrt{B} \sqrt{1-a(s)}\left(e^{-B^{2}[a(s)-1]^{2} / 2}-e^{-B^{2} / 2}\right),
\end{gathered}
$$

and we set $\phi_{D}(r, z, 0)=0$. The initial geometry for $B=5$ and $C=2$, referred to as geometry 1 , is depicted in Fig. 2. Drop geometry 2 was built by making the left side of the previous geometry symmetric with respect to $z=3.5$ as is shown in Fig. 3.

\section{B. Level set numerical schemes}

The fixed computational domain for Eqs. (19) and (20) is taken as $\Omega_{D}=\left[0, L_{1}\right] \times\left[0, L_{2}\right], L_{1}, L_{2}$ being such that $\Omega_{D}$ will contain the free boundary for all $t \in[0, T]$ (see Fig. 2). A rectangular mesh over the domain $\Omega_{D}$ defines a set of points $D_{\Delta}=\left\{\left(r_{i}, z_{j}\right): r_{i}=i \Delta r, z_{j}=j \Delta z, i=1, N, j=\right.$ $1, M\}$, with $N, M$ the number of mesh points in the $r$ and $z$ directions and $\Delta r, \Delta z$ the corresponding mesh sizes. Let $\mathbf{n}=\left(n_{r}, n_{z}\right)$ be the unit normal vector to $\Gamma_{t_{n}}(s)$ and $u, v$ the radial and axial inner fluid velocity components. The axisymmetric assumption implies $u=0$ and $n_{r}=0$ at $\Gamma_{z}$, and thus

$$
\frac{\partial \Psi^{n}}{\partial r}=0 ; \quad \frac{\partial G^{n}}{\partial r}=0 \text { at } \Gamma_{z}
$$

${ }^{1}$ These formulas were kindly supplied by Professor David Leppinen.
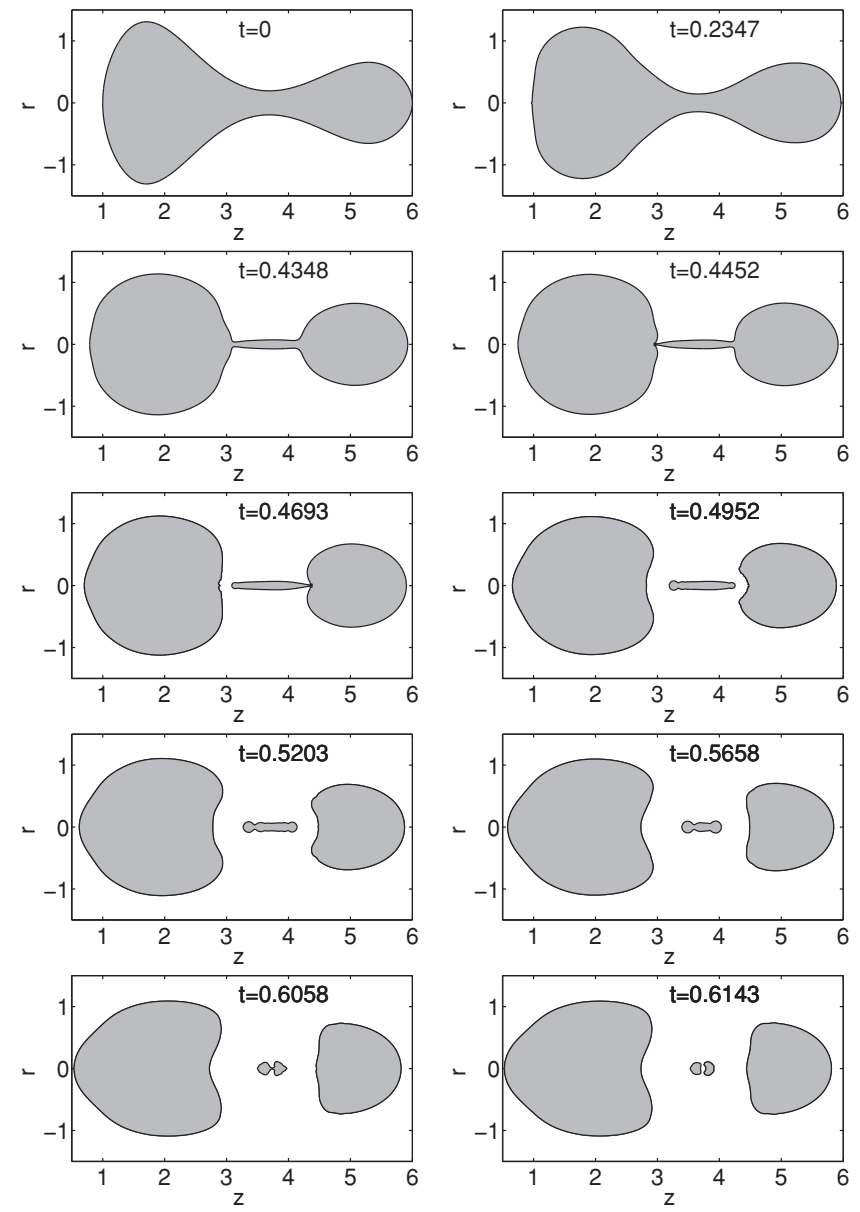

FIG. 11. Front profiles at indicated times, $D=0$.

will be imposed for Eqs. (19) and (20). Let $G_{i, j}^{n}$ be the numerical approximation of the fictitious potential $G\left(r_{i}, z_{j}, t_{n}\right)$. A first-order upwind scheme approximation of Eq. (20) yields, for $i=2, N-1 ; j=2, M-1$,

$$
\begin{aligned}
G_{i, j}^{n+1}= & G_{i, j}^{n}-\Delta t\left[\max \left(u_{i, j}^{n}, 0\right) D_{i, j}^{-r}+\min \left(u_{i, j}^{n}, 0\right) D_{i, j}^{+r}\right. \\
& \left.+\max \left(v_{i, j}^{n}, 0\right) D_{i, j}^{-z}+\min \left(v_{i, j}^{n}, 0\right) D_{i, j}^{+z}\right]+\Delta t f_{i, j}^{n},
\end{aligned}
$$

where

$$
\begin{gathered}
D_{i, j}^{-r}=D_{i, j}^{-r}\left\{G_{i, j}^{n}\right\}=\frac{G_{i, j}^{n}-G_{i-1, j}^{n}}{\Delta r}, \\
D_{i, j}^{+r}=D_{i, j}^{+r}\left\{G_{i, j}^{n}\right\}=\frac{G_{i+1, j}^{n}-G_{i, j}^{n}}{\Delta r}
\end{gathered}
$$

are the backward and forward finite difference approximations for the derivative in the radial direction (the same expressions hold for the corresponding $z$ derivatives $D_{i, j}^{-z}$ and $D_{i, j}^{+z}$ ). The discrete boundary conditions are

$$
\begin{gathered}
v_{1, j}=0 \quad \text { for } j=1, M, \\
\frac{\partial G_{i, j}^{n}}{\partial r} \approx \frac{4 G_{2, j}^{n}-3 G_{1, j}^{n}-G_{3, j}^{n}}{2 \Delta r} \quad \text { for } \quad\left(r_{i}, z_{j}\right) \in \Gamma_{z}, \\
G_{i, 1}^{n}=G_{i, 2}^{n} ; G_{i, M-1}^{n}=G_{i, M}^{n} \quad \text { for } \quad i=1, N, \\
G_{N, j}^{n}=G_{N-1, j}^{n} ; G_{1, j}^{n}=G_{2, j}^{n} \quad \text { for } \quad j=1, M .
\end{gathered}
$$

The same discrete equations, without a source term, can be written for $\Psi$, Eq. (19). 

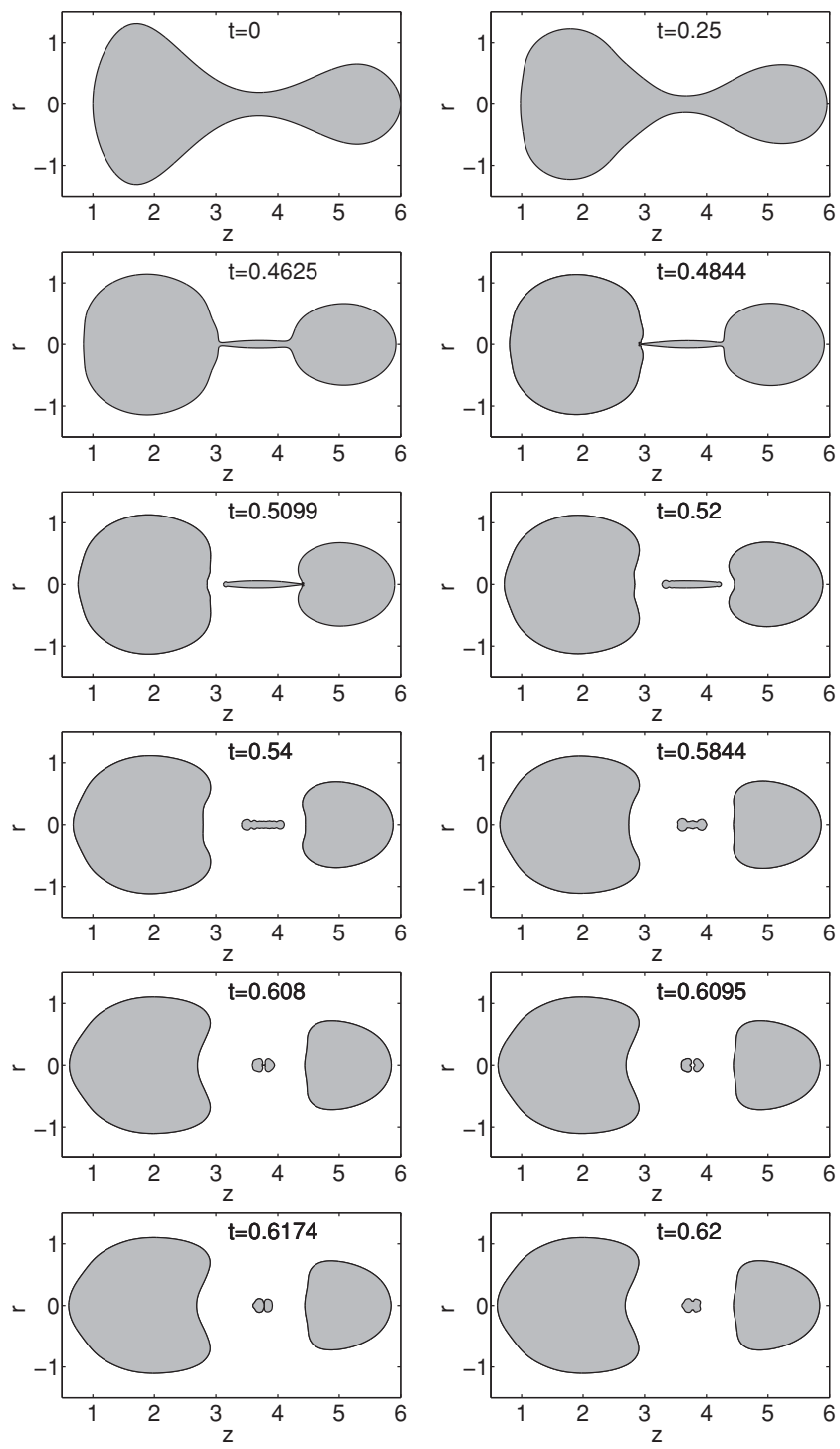

FIG. 12. Front profiles at indicated times, $D=0.3$.

Note that, for simplicity, we have written $u, v, f$ instead of $u_{\mathrm{ext}}, v_{\mathrm{ext}}, f_{\mathrm{ext}}$, and we describe a first-order explicit scheme with a centered source term. Initial values of $G_{i, j}^{0}$ are obtained by extending $\left.\phi_{D}(r, z, 0)\right|_{\Gamma_{0}(s)}$. However, at any time step $n$ it is always possible to perform a new extension of $\phi_{D}^{n}(r, z, n \Delta t)$ and a reinitialization of the level set function. We remark here that if reinitialization is done too often, especially using poor reinitialization techniques, spurious mass loss or gain will occur. Thus it is important to perform reinitialization both sparingly and accurately. For the calculations reported below, conservation of total volume and energy are monitored, and these numbers are seen to be constant within reasonable accuracy. The relative error in volume and energy are less than $0.2 \%$, respectively.

A key issue is how one obtains $\mathbf{u}_{\mathcal{I}_{\text {ext }}}$ and $f_{\text {ext }}$ on the grid points of $\Omega_{D}$. One is free to choose any extension for the velocity and the right-hand side, as long as they smoothly tend to the correct values on the interface, and do not induce instabilities in the resulting flow. Given any point in the domain, a natural way to construct such an extension is to
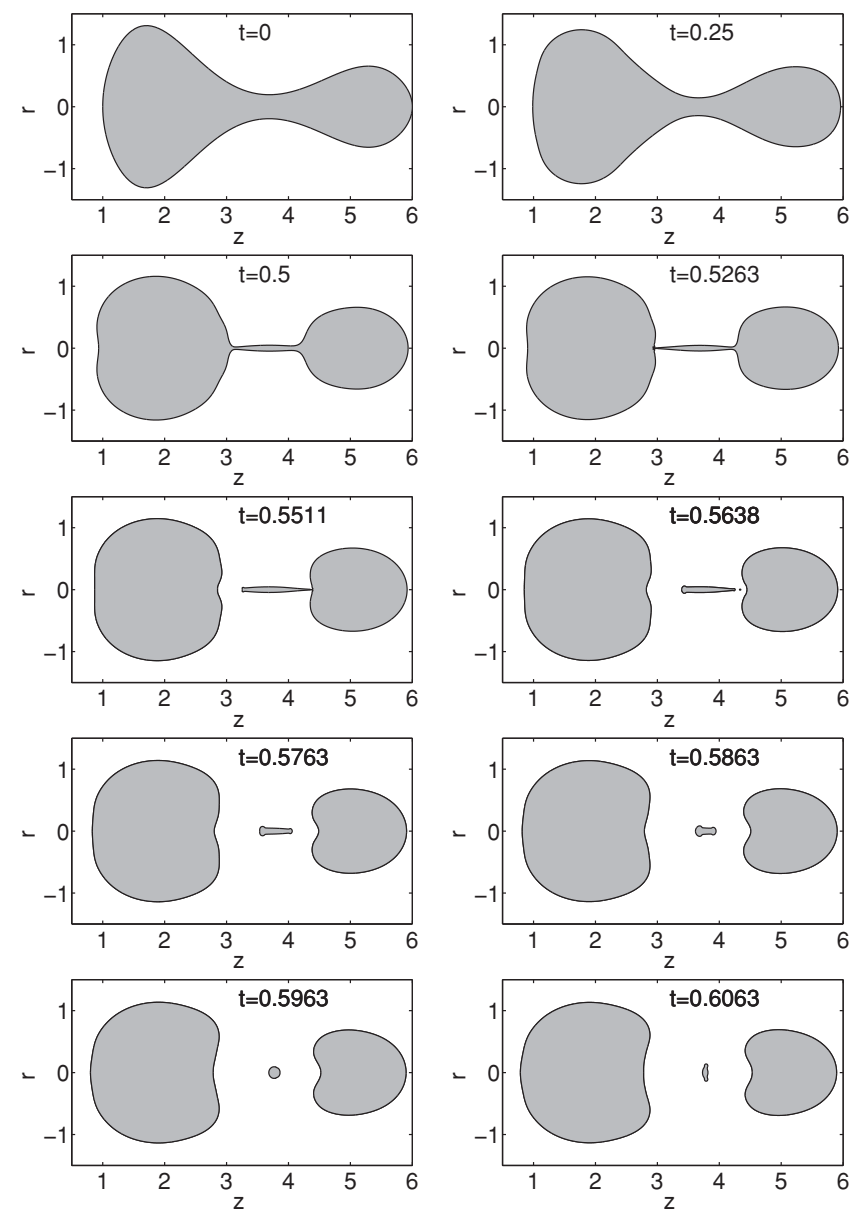

FIG. 13. Front profiles at indicated times, $D=1$.

choose the value at this point to be the same as that of the closest point on the interface: this idea was first introduced in [15], and executed by following the characteristics of the signed distance function corresponding to the level set function. An equivalent formulation is to solve the equation $\nabla W \cdot \nabla \Psi=0$, where $W$ is the quantity to be extended: This was discussed in detail in [13], along with a fast Dijkstra-like method to solve this equation. In our case, we calculate $f$ on free surface nodes, and use these values together with the condition $\nabla f \cdot \nabla \Psi=0$ to obtain $f_{\text {ext }}$. For the inner fluid velocity components we follow the same strategy, $\nabla u \cdot \nabla \Psi=0, \nabla v \cdot \nabla \Psi=0$. This algorithm for extending quantities defined on the front to off the front works very well for the velocity field in the case of Eq. (15), as it maintains the signed distance function for the level sets of $\Psi$.

\section{Boundary integral equations}

The direct boundary integral formulation for the two-fluid problem that is employed in this work has been discussed in detail in [11], and thus this section provides only a brief summary of this approach. The method is direct in that, in contrast to previous work [6,7], the potentials and normal derivatives for the two fluids are the basic variables. It is also worth noting that there are other significant differences with the boundary integral approximations employed in $[6,7]$. Herein, a linear element Galerkin method [16] is employed, in 

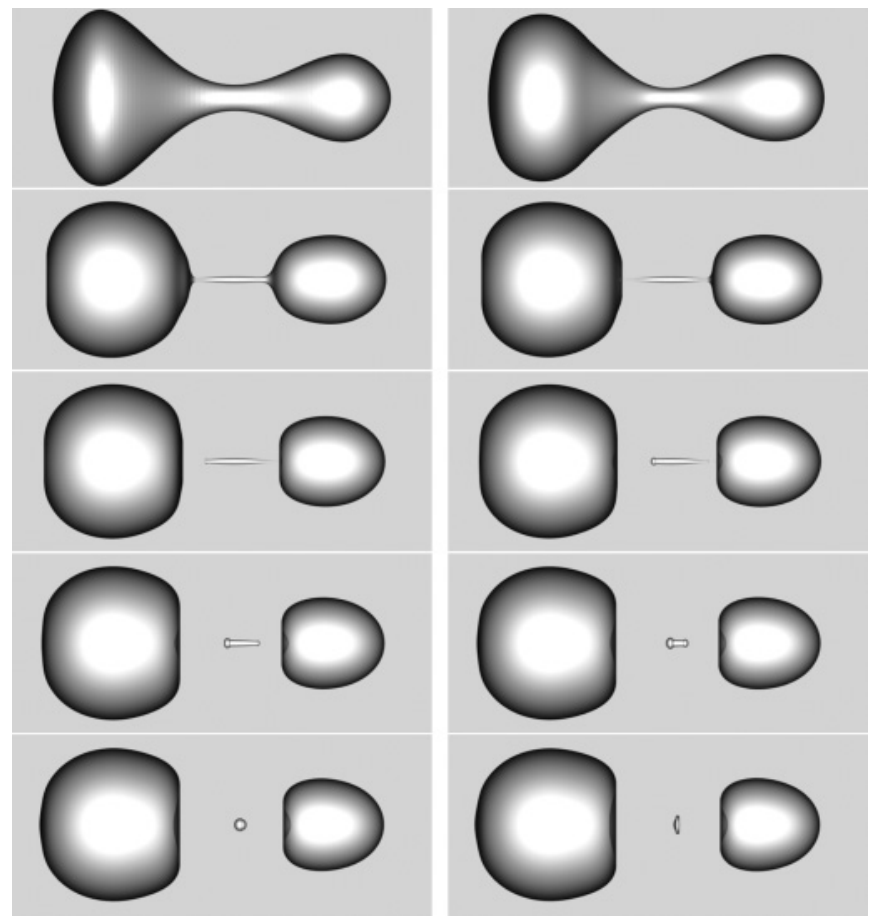

FIG. 14. 3D Front profiles at various times (same as in Fig. 13), $D=1$. For the horizontal axis $0.4 \leqslant z \leqslant 6.6$ and the vertical axis $-1.33 \leqslant r \leqslant 1.33$.
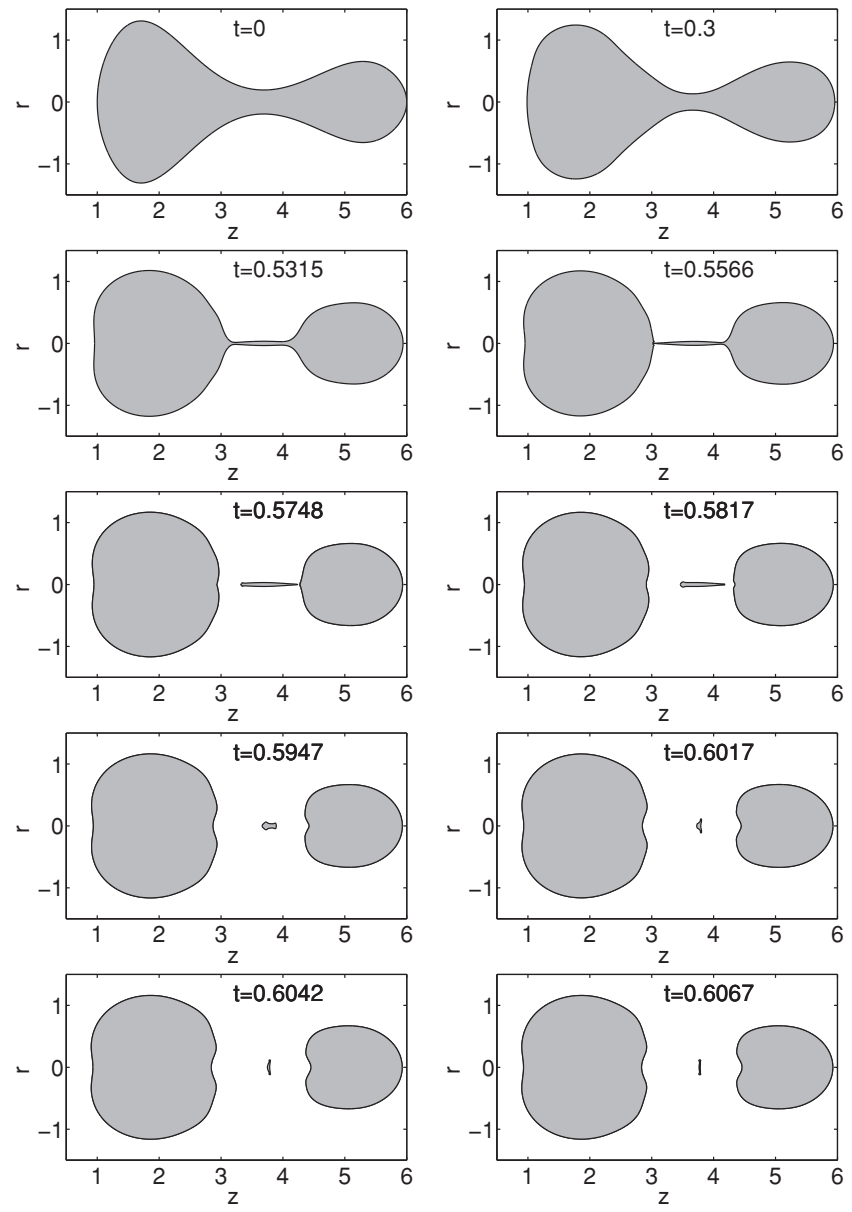

FIG. 15. Front profiles at indicated times, $D=2$.
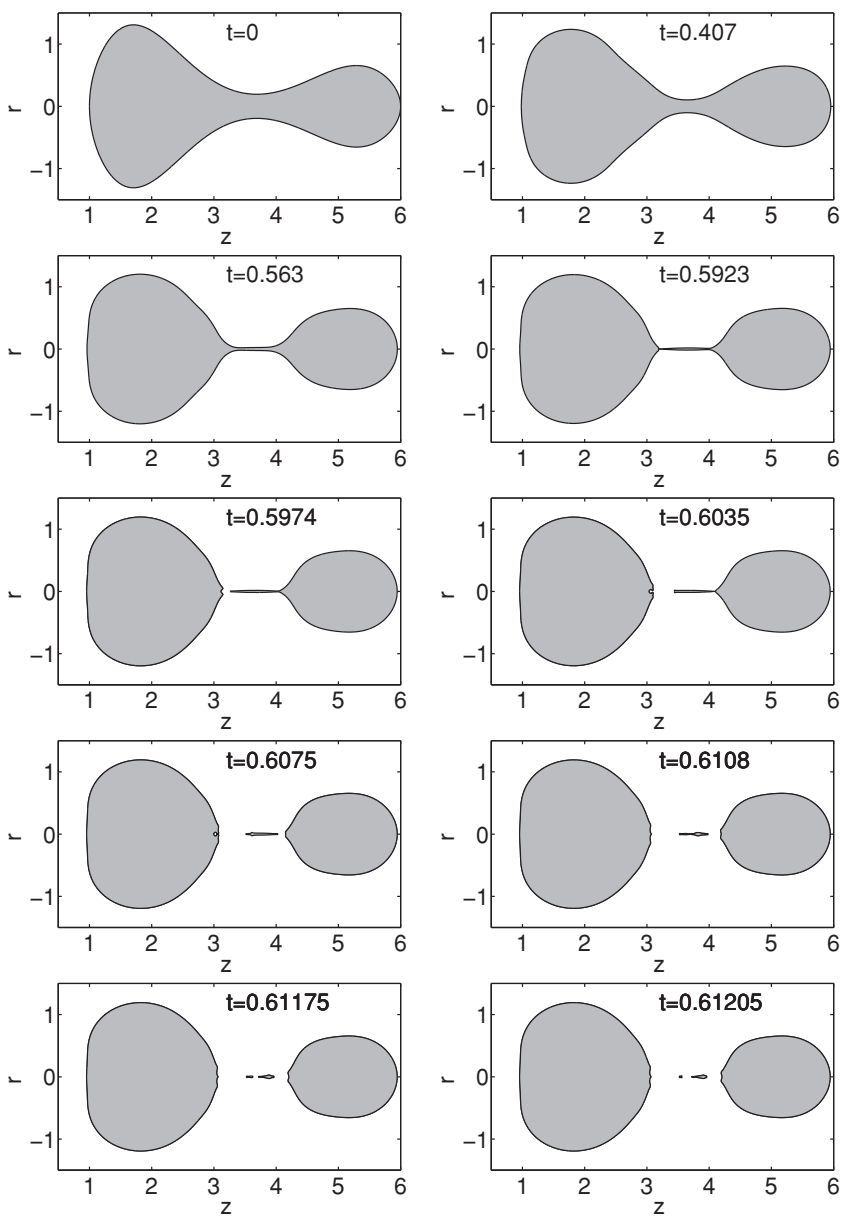

FIG. 16. Front profiles at indicated times, $D=4$.

contrast to the high-order collocation approximation in [6,7], and the methods for computing the critical surface gradient are completely different.

With the linear element discretization and $N_{p}$ free boundary nodes, the boundary integral equations for the exterior and interior fluids, Eqs. (14), become the matrix equations

$$
\begin{aligned}
& \mathcal{H}_{\mathcal{E}} \phi_{\mathcal{E}}-\mathcal{G}_{\mathcal{E}} \frac{\partial \phi_{\mathcal{E}}}{\partial \mathbf{n}}=0, \\
& \mathcal{H}_{\mathcal{I}} \phi_{\mathcal{I}}-\mathcal{G}_{\mathcal{I}} \frac{\partial \phi_{\mathcal{I}}}{\partial \mathbf{n}}=0 .
\end{aligned}
$$

This can be simplified by directly incorporating the interface conditions: The interior and exterior potentials are related through the prescribed interface function $\phi_{D}=\phi_{\mathcal{I}}-D \phi_{\mathcal{E}}$, and the fluid normal derivatives differ only in sign. Moreover, an examination of the boundary integral equations shows that $\mathcal{G}_{\mathcal{E}}=\mathcal{G}_{\mathcal{I}}$ and that $\mathcal{H}_{\mathcal{I}}$ and $\mathcal{H}_{\mathcal{E}}$ differ by a sign except for the singular term that is discontinuous crossing the boundary. Defining $\hat{\mathcal{H}}_{\mathcal{E}}=-\mathcal{H}_{\mathcal{I}}, \hat{\mathcal{H}}_{\mathcal{E}}$ will differ from $\mathcal{H}_{\mathcal{E}}$ only in the discontinuous jump term. Incorporating this information, one can eliminate the interior fluid quantities in Eq. (21) to obtain the system

$$
\left(\begin{array}{cr}
\mathcal{H}_{\mathcal{E}} & -\mathcal{G}_{\mathcal{E}} \\
-D \hat{\mathcal{H}}_{\mathcal{E}} & \mathcal{G}_{\mathcal{E}}
\end{array}\right)\left(\begin{array}{c}
\phi_{\mathcal{E}} \\
\frac{\partial \phi_{\mathcal{E}}}{\partial \mathbf{n}}
\end{array}\right)=\left(\begin{array}{c}
0 \\
\hat{\mathcal{H}}_{\mathcal{E}} \phi_{D}
\end{array}\right)
$$



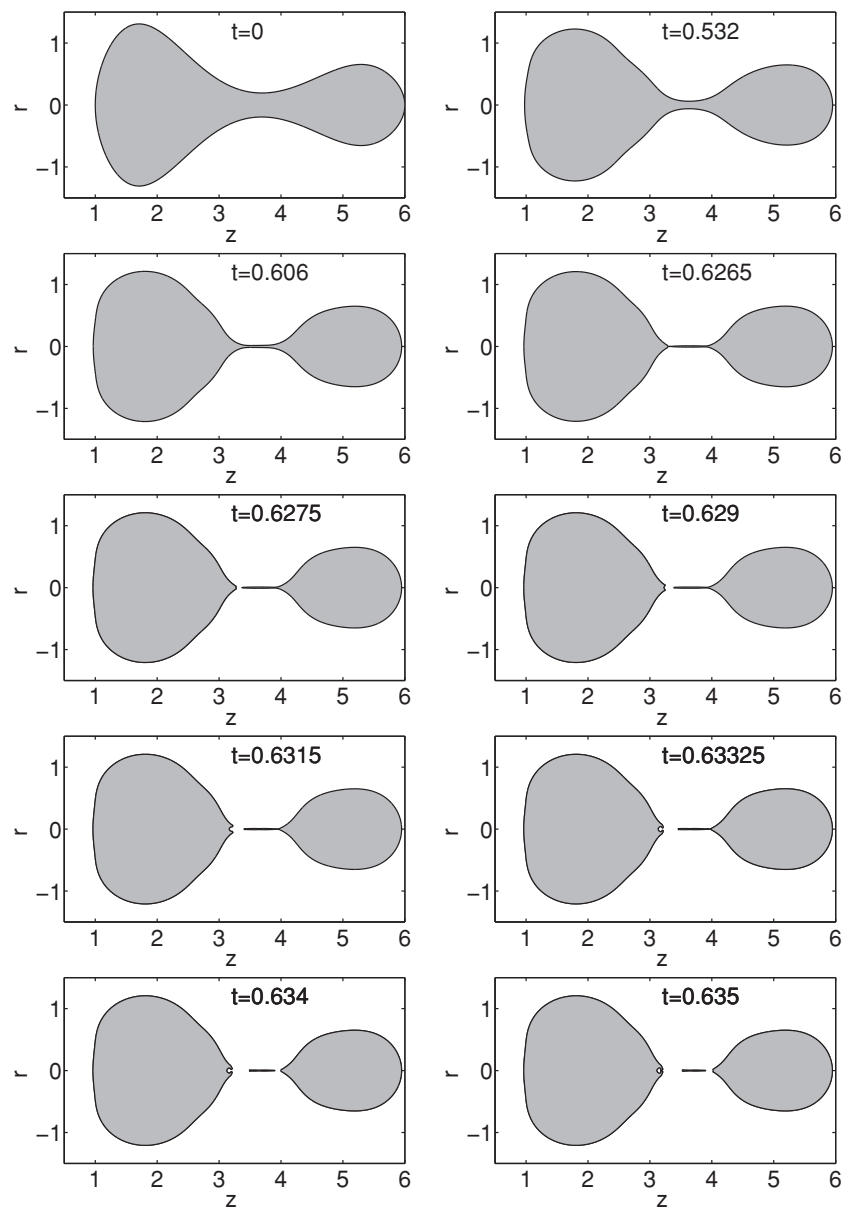

FIG. 17. Front profiles at indicated times, $D=6$.

Finally, this $2 N_{p} \times 2 N_{p}$ set of equations can be further simplified to solving two-order $N_{p}$ matrix systems,

$$
\begin{gathered}
\left(\mathcal{H}_{\mathcal{E}}-D \hat{\mathcal{H}}_{\mathcal{E}}\right) \phi_{\mathcal{E}}=\hat{\mathcal{H}}_{\mathcal{E}} \phi_{D}, \\
\mathcal{G}_{\mathcal{E}} \frac{\partial \phi_{\mathcal{E}}}{\partial \mathbf{n}}=\mathcal{H}_{\mathcal{E}} \phi_{\mathcal{E}}
\end{gathered}
$$

Moreover, with the Galerkin scheme described in [16] the second subsystem is symmetric. Consequently, the construction of the two-fluid boundary integral matrices is no more expensive than for a single fluid. However, compared to a single fluid simulation, the two-fluid solution involves an additional matrix-verctor multiplication to compute $\mathcal{H}_{\mathcal{E}} \phi_{\mathcal{E}}$ and an additional resolution of a $N_{p} \times N_{p}$ symmetric linear system. Once the exterior boundary functions have been obtained from Eq. (22), the interior functions are known as well, and the algorithm discussed in [16] can be applied in each fluid to obtain the needed boundary gradients.

\section{Free surface numerical representation}

In a level set formulation the position of the front, $\Psi=0$, is extracted from the level set nodal values of the fixed grid, $\Omega_{D}$. Here we use a first order linear approximation of the free surface, yielding a polygonal interface formed by unevenly distributed nodes, which are the level set nodes. At each time step, to overcome instabilities due to very close nodes for the boundary element calculation and to reduce computational
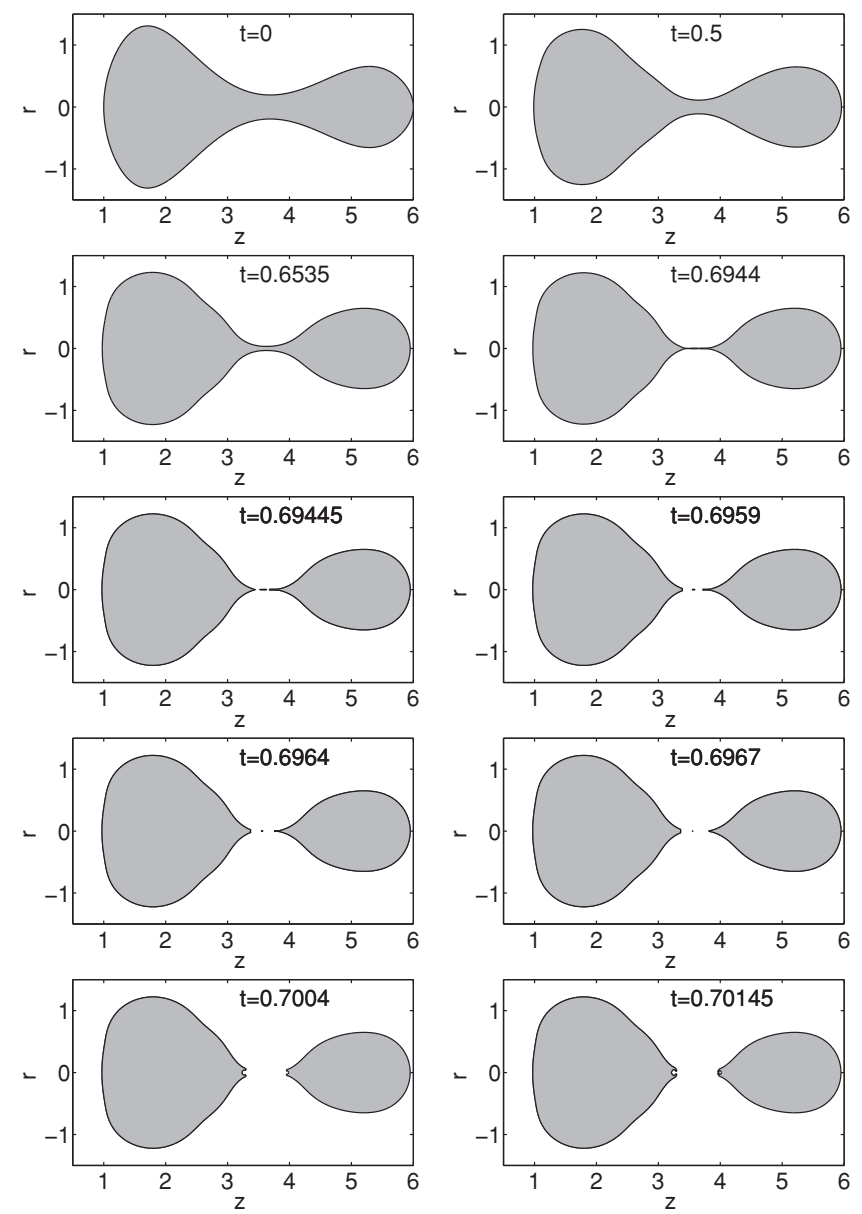

FIG. 18. Front profiles at indicated times, $D=10$.

time, a regriding technique described in [8] has been employed. Here the new set of nodes $N_{p}$, used for the boundary integral calculation and the level set extensions, are equally spaced giving a constant boundary mesh size $\Delta s$. These reduced set of nodes will be referred to as BEM nodes.

\section{NUMERICAL RESULTS}

In order to validate the computed results obtained with our model, all the standard numerical tests required to this end have been carried out. In particular, a complete numerical analysis comparing the numerical results with the analytical solution of an oscillating sphere was presented in [11]. In what follows we present the numerical experiments and results for the breakup and post breakup dynamics of a two lobe geometry drop.

\section{A. Two-fluid system breakup simulations}

A set of numerical experiments have been carried out for different $D$ values, starting always with the initial conditions described in Sec. IV A, geometry 1. The computations were also carried out after pinchoff to study post separation dynamics and the evolution, if present, of the satellite drops. The fixed domain for the level set computations is set to $\Omega_{D}=[-2,2] \times[0,8]$. As the flow is much faster after first pinchoff event and very tiny structures may appear, we have organized the numerical experiments before and after pinchoff. 

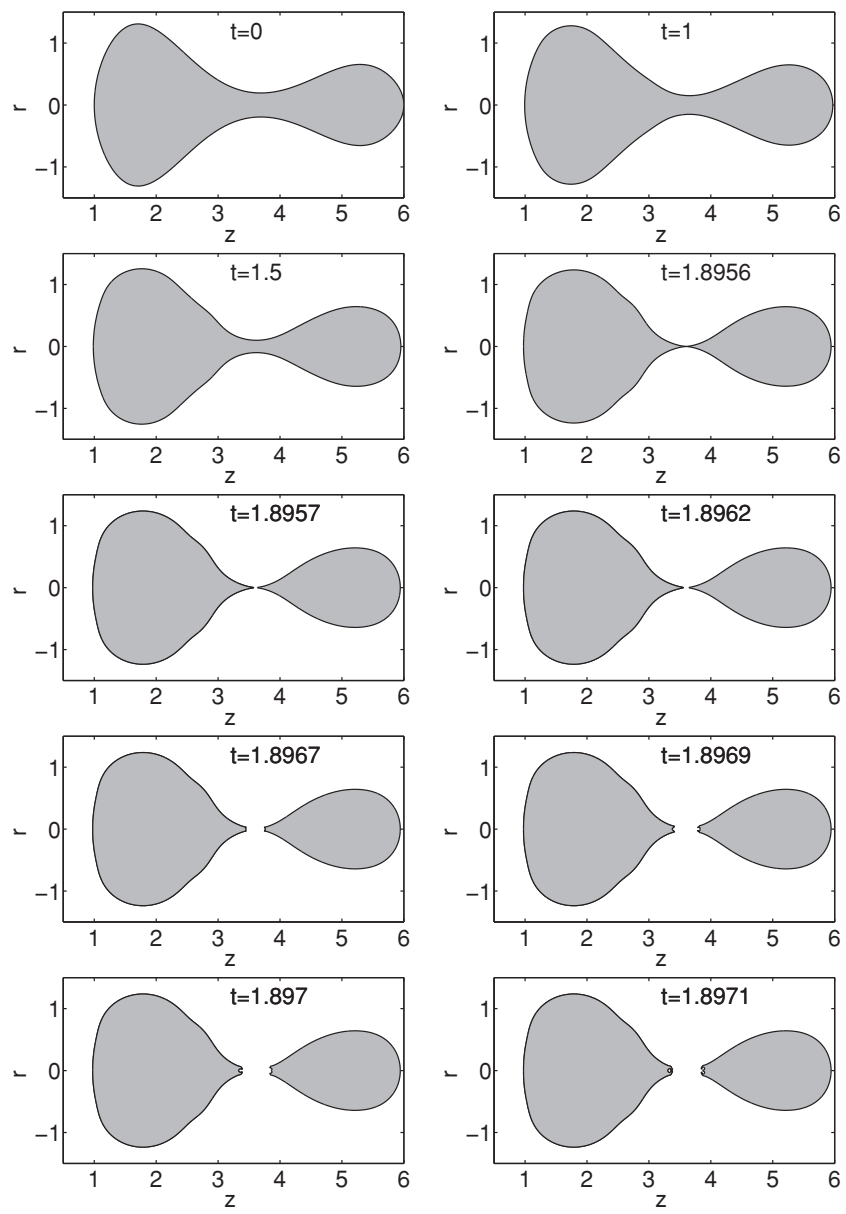

FIG. 19. Front profiles at indicated times, $D=100$.

\section{Before breakup results}

To check the behavior of the computed solution with respect to the discretization parameters we have run two cases: (a) $\Delta r=\Delta z=0.01, \quad N_{p}=201$, and (b) $\Delta r=\Delta z=$ $0.005, N_{p}=301$, which will be referred to as coarse and fine grids. As the total arc length of the free boundary changes with time, the boundary mesh size also changes, being $\Delta s \approx 0.033$ and $\Delta s \approx 0.025$ for cases (a) and (b), respectively. The time step is 0.001 initially and changed adaptively according to the scheme described in [11].

The variables used to characterize the interior fluid flow are the evolution of the minimum neck radios $r_{\min }$ with time, the nondimensional pinchoff time $t_{p}$, the relative error in the
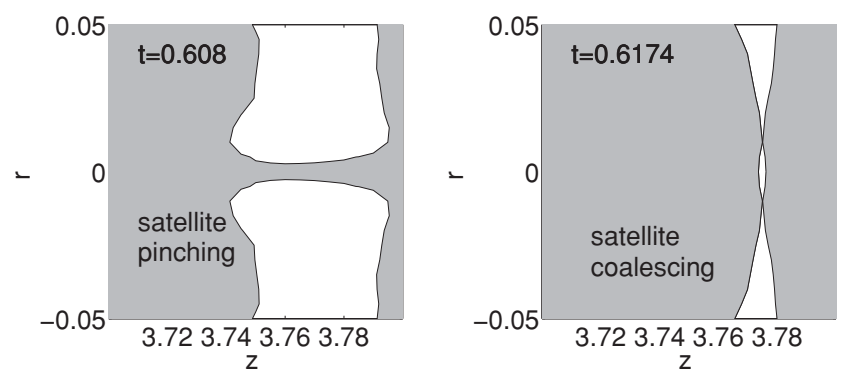

FIG. 20. Focused details of satellite pinching and coalescing at indicated times, $D=0.3$.

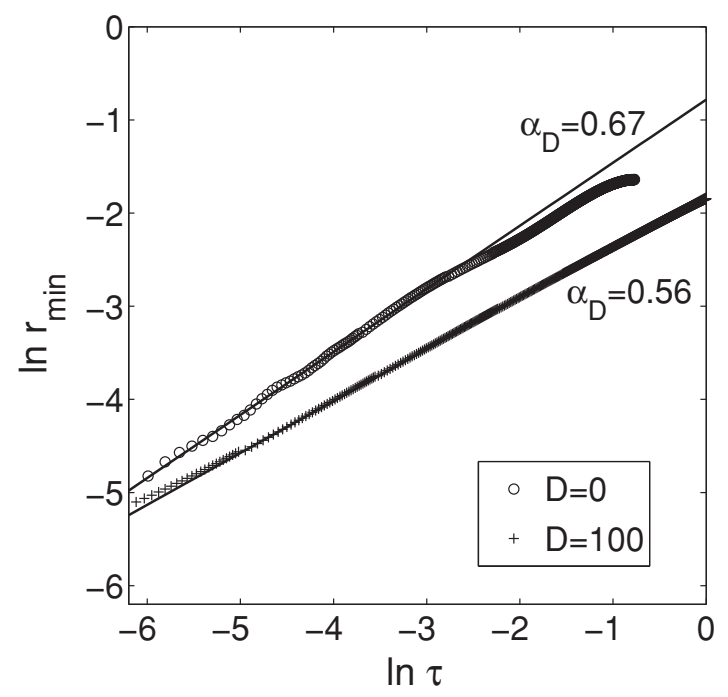

FIG. 21. Scaling of $r_{\min }$ at pinchoff for $D=0$ and $D=100$.

volume of the inner fluid $e_{V}$, and the pinchoff profiles near breakup. In Fig. 4 we show the values of $r_{\min }$ versus time for both mesh sizes and parameter values $D=0,1,2,4,6,20,100$, and in Table I nondimensional pinchoff time and relative error in drop volume are listed for all $D$ values and both grids.

The evolution of $r_{\min }$ versus time is almost the same for both meshes, although the breakup happens slightly earlier using the finer grid for all $D$ values. This could be due to the fact that numerical viscosity diminishes with grid size. Note that the nondimensional pinchoff time increases with $D$, but when converting it to real time, multiplying by the appropriate $t_{0}$ for each case, it actually diminishes as $D$ increases, in accordance with the physical evidence that bubbles break up sooner than droplets.

The relative error in the inner drop volume is always less than $0.15 \%$, and also diminishes by increasing the grid resolution. Figure 5 shows the front profiles focused near the pinchoff region for $D=0,1,4,6,10,20$. When $0 \leqslant D \leqslant 1$ the overturning of the profile before separation is quite

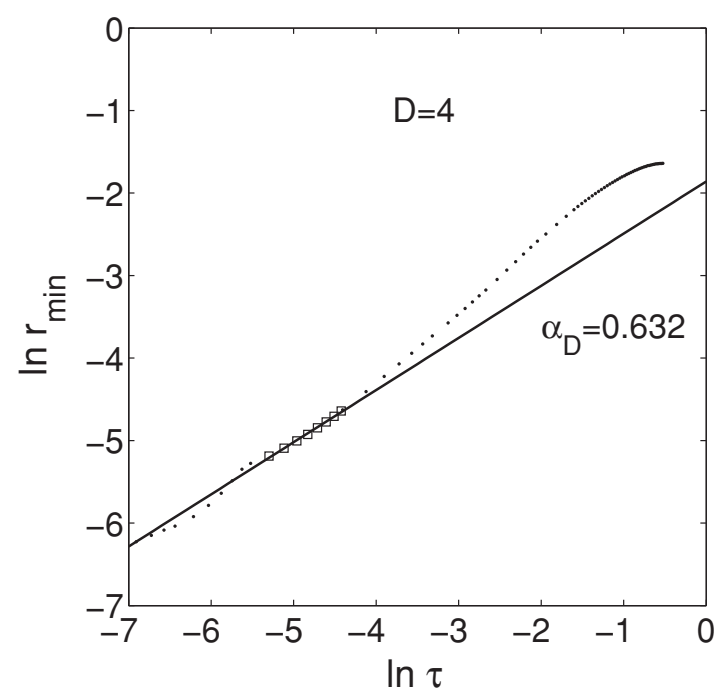

FIG. 22. Scaling of $r_{\min }$ for $D=4$ (squares for the fitted region). 


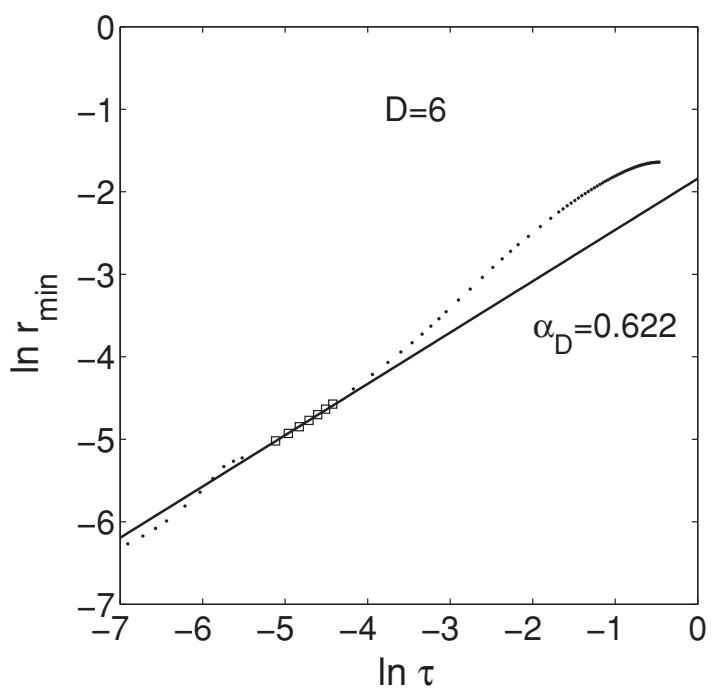

FIG. 23. Scaling of $r_{\min }$ for $D=6$ (squares for the fitted region).

pronounced, whereas for $D>4$ there is no overturning. Moreover, the numerical instabilities for large $D$ seen in previous work are absent. To show that this is not due to the mesh resolution we depict in Fig. 6 the coarse and fine profiles at pinchoff time for $D=6$ which are nearly indistinguishable. In Figs. 7 and 8 we show the profiles for both meshes focused near the pinchoff point for $D=4$ and $D=6$, respectively. Here the level set nodes are marked. As expected, the finer grid better resolves the front details, with the relative difference in the axial coordinate at pinchoff time less than $2 \%$ for all $D$ values.

\section{After breakup results}

In the case of drop geometry 1 , with the initial neck separating two nonequal fluid masses, the satellite drop appears at the second pinchoff event for $D=0,0.3,1,2,4,6$. The satellite size diminishes and evolves faster as $D$ increases. To follow the evolution of such tiny drops $\Delta r=\Delta z$ and $\Delta s$ should be small enough, restricting the time step size accordingly. The discretization parameters are set to $\Delta r=\Delta z=0.005$ and $N_{p}=201$ for each of the two main drops, which makes $\Delta s \approx 0.015-0.02$. For the satellite drop, depending upon its total arc length, we take $\Delta s \approx 0.005-0.01$. Satellites with sizes under the mesh resolution cannot be resolved and are automatically removed from the simulation.

TABLE III. Computed $\alpha_{D}, V_{D}, t_{p}$, and $e_{V}$ for geometry 1 .

\begin{tabular}{lcccc}
\hline \hline$D$ & $\alpha_{D}$ & $V_{D}$ & $t_{p}$ & $e_{V}$ \\
\hline 0 & 0.670 & $1.510834 \times 10^{-1}$ & 0.4551 & $1.4499 \times 10^{-3}$ \\
0.3 & 0.660 & $1.062827 \times 10^{-1}$ & 0.4844 & $1.0496 \times 10^{-3}$ \\
1 & 0.651 & $5.162887 \times 10^{-2}$ & 0.5263 & $5.5482 \times 10^{-4}$ \\
2 & 0.642 & $2.128503 \times 10^{-2}$ & 0.5566 & $4.6596 \times 10^{-4}$ \\
4 & 0.632 & $4.668228 \times 10^{-3}$ & 0.5923 & $4.6892 \times 10^{-4}$ \\
6 & 0.622 & $1.347971 \times 10^{-3}$ & 0.6265 & $4.1447 \times 10^{-4}$ \\
10 & 0.593 & $1.049214 \times 10^{-4}$ & 0.6944 & $2.7416 \times 10^{-4}$ \\
20 & 0.572 & 0 & 0.8620 & $2.2308 \times 10^{-4}$ \\
100 & 0.560 & 0 & 1.8956 & $1.8306 \times 10^{-4}$ \\
\hline \hline
\end{tabular}

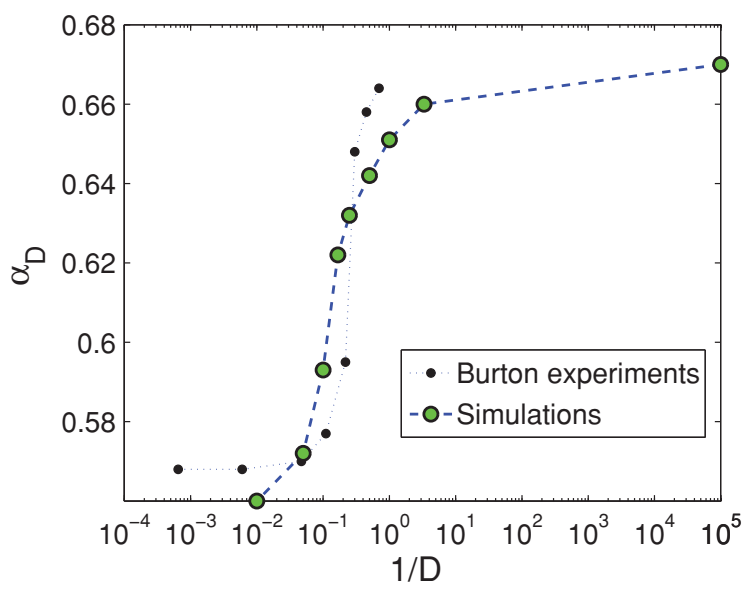

FIG. 24. (Color online) Scaling exponents versus $D$, calculated and experimental values, geometry 1 .

The total simulated time is different for each $D$ value, as the time step should be diminished from $\Delta t=5 \times 10^{-5}$ for $D=0,0.3,1,2,4$ to $\Delta t=10^{-5}$ for $D=6,10,20,100$. Moreover, for these larger $D$ values the exterior fluid penetrates completely the interior fluid, at which point the simulation is stopped. See Fig. 9 at $t=0.635$ for $D=6$, in which the BEM nodes are also depicted. At this particular time the number of nodes for the satellite drop is $N_{p}=79$. The same pattern is seen in Fig. 10 for $D=100$ at $t=1.89714$. This behavior could indicate the initiation of a Worthington jet as reported in [17].

In Table II we show for each $D$ value the second pinchoff time $t_{s}$, the final time $t_{f}$, the relative error in the inner fluid volume at $t_{f}, e_{V}$, and the total number of time steps performed, $N_{\text {steps }}$.

Volume conservation of the inner fluid is very accurate, as the volume lost is always less than $0.25 \%$ at the end of each simulation. Finally, in Figs. 11, 12, 13, 14, 15, 16, 17, 18 , and 19 the complete evolution of the two-fluid system, before and after pinchoff time, at the indicated times, is depicted for $D=0,0.3,1,2,4,6,10,100$, respectively. Note that the pictures in Fig. 14 are 3D renderings of the 2D profiles for $D=1$ shown in Fig. 13. We include them to enhance the sense of reality and to be able to compare the structures of the computed satellite drop with those seen in laboratory experiments [2,5]. It is also possible to visualize the complete simulation corresponding to $D=1$ in the supplemental material [18]. By inspecting the numerical profiles, it can be seen that not only does the shape at separation depend strongly upon the density ratio, but also the evolution and shapes of the main drops and satellites. For $D<1$

TABLE IV. Computed $\alpha_{D}, V_{D}, t_{p}, e_{V}$, and $e_{E}$ for geometry 2 .

\begin{tabular}{cccccc}
\hline \hline$D$ & $\alpha_{D}$ & $V_{D}$ & $t_{p}$ & $e_{V}$ & $e_{E}$ \\
\hline 0 & 0.678 & $9.0251 \times 10^{-4}$ & 0.63823 & $2.4830 \times 10^{-3}$ & $1.4927 \times 10^{-3}$ \\
1 & 0.659 & $1.5225 \times 10^{-4}$ & 0.72132 & $9.4134 \times 10^{-4}$ & $1.3795 \times 10^{-3}$ \\
2 & 0.640 & $5.3837 \times 10^{-5}$ & 0.79310 & $8.9296 \times 10^{-4}$ & $2.3295 \times 10^{-3}$ \\
4 & 0.599 & 0 & 0.88755 & $7.7861 \times 10^{-4}$ & $1.4714 \times 10^{-3}$ \\
6 & 0.585 & 0 & 0.97710 & $7.3217 \times 10^{-4}$ & $3.8314 \times 10^{-4}$ \\
\hline \hline
\end{tabular}




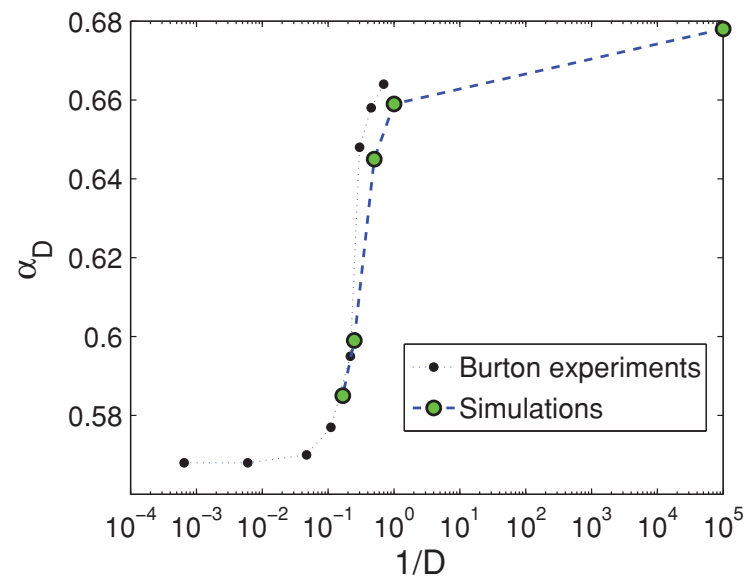

FIG. 25. (Color online) Scaling exponents versus $D$, calculated and experimental values, geometry 2 .

tiny capillary waves propagate through the surface of the satellite drop leading to subsequent pinchoff events. For $D=0$ and $D=0.3$ the satellites break up at times $t=0.6081$ and $t=0.60825$, respectively, showing profile overturning before pinchoff, but soon after the two smaller satellites coalesce entrapping a microbubble; see focused details of these events for $D=0.3$ in Fig. 20 .

For $1 \leqslant D \leqslant 4$ the satellite is compressed by the exterior fluid, evolving quickly toward a spherical shape, but is then further elongated to a narrow disc; see Fig. 13. Values in the range $4<D \leqslant 10$ lead to filamentlike satellites, which eventually split into smaller satellites. In particular, for $D=10$ a very tiny inner fluid drop develops at separation following the same pattern as predicted by [19]; see Fig. 18 at time $t=0.69445$. For $D \geqslant 20$ no satellites are present, at least within the space and time resolution of the present simulations.

\section{B. Transition regime from droplet-to-bubble behavior}

To investigate the droplet $(D=0)$-to-bubble $(D \rightarrow \infty)$ transition, the computations were initiated with the same "two-lobe" drop geometry 1 , and values $D=$ $0,0.3,1,2,4,6,10,20,100$ were selected. All the computations before breakup have been performed with two different sets of discretization parameters as explained previously. In this

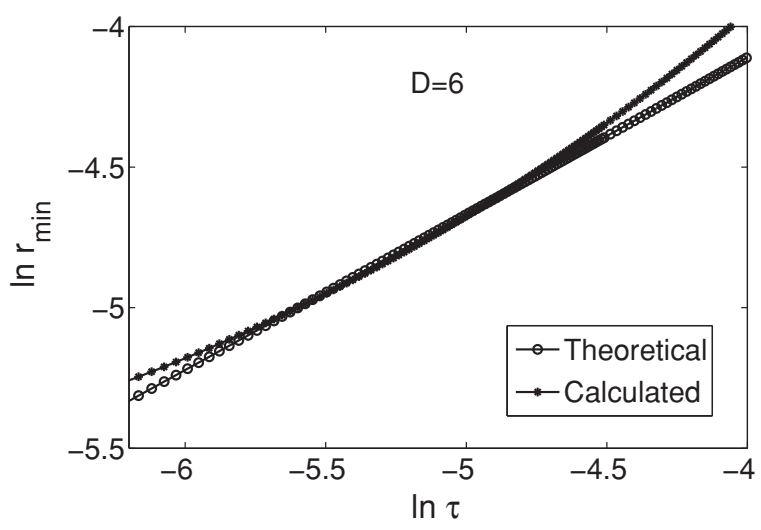

FIG. 26. $r_{\text {min }}$ evolution, theoretical and calculated, for $D=6$ and geometry 2 .
TABLE V. Calculated scaling exponents depending upon $r_{\min }$ range for $D=6$ (geometry 2 ).

\begin{tabular}{lc}
\hline \hline$r_{\text {min }}$ Range & $\alpha_{D}$ \\
\hline$[0.0066,0.0121]$ & 0.585 \\
{$[0.0061,0.0121]$} & 0.572 \\
{$[0.0061,0.0112]$} & 0.56 \\
\hline \hline
\end{tabular}

section the reported scaling exponents are based upon results for the finer mesh.

It is generally accepted that the solution of the two-fluid system approaching pinchoff time is self-similar for any $D$ and the minimum neck radius follows a power law

$$
r_{\min } \propto \tau^{\alpha_{D}}
$$

where $\tau=t_{p}-t$ is the remaining time until pinchoff. For $D=0$, droplets in air, $\alpha_{D}=2 / 3$ [6] and for $D=\infty$, i.e., an air bubble, the scaling exponent $\alpha_{\infty}$ is not constant but rather has a logarithmic correction

$$
\alpha(\tau) \approx \frac{1}{2}+\frac{1}{4 \sqrt{-\ln \tau}}
$$

and the approach to the asymptotic limit $\frac{1}{2}$ is exceedingly slow [20]. Detailed experiments yield an approximate effective exponent with $\alpha_{\infty} \approx 0.53-0.57$ [2,21]. Recently, for air bubbles, it has been reported [22] that the time scale for the onset of this universal final regime, characterized by the above exponent, is found to vary by orders of magnitude depending on the various physical realizations, from the milliseconds range to the microseconds range.

Calculated scaling results for the two extremes, $D=0$ and $D=100$, are shown in Fig. 21, which plots $\ln r_{\text {min }}$ versus $\ln \tau$. The linear fit yielded 0.66 and 0.56 for the computed power law exponents, in excellent agreement with known results.

For intermediate values of $D$, the evaluation of the exponents is more difficult. As seen in the scaling plot for $D=4$ and $D=6$, Figs. 22 and 23, the linear region is shorter and the value for $\alpha_{D}$ will vary somewhat depending upon the chosen interval. We have taken nondimensional values in the range $0.005<r_{\min }<0.011$, which corresponds approximately to the same fitting interval reported in [5]. To make Figs. 22 and 23 clearer only one out of ten data points have been plotted. Nevertheless, we have observed that the calculated exponents for these intermediate $D$ values diminish as we get closer to pinchoff, which is consistent with the slowly decreasing formula (24).

The computed exponents $\alpha_{D}$, the satellite drop volume (relative to the total volume in $\%$ ) $V_{D}$, the pinchoff time $t_{p}$, and and the relative error in computed volume at pinchoff time $e_{V}$, for the finer grid are listed in Table III. Note that the nondimensional pinchoff time increases with $D$, but actually when converted to real time we get $76 \mathrm{~ms}$ for water droplets and $7.9 \mathrm{~ms}$ for air bubbles.

In Fig. 5 we show the $2 \mathrm{D}$ front profiles for $D=$ $0,1,4,6,10,20$ at pinchoff time. The overturning of the fluid at pinchoff present in Fig. 5 for $D=0,1$ is already absent for $D=6$. Moreover, for $D=6$, the overturning and interface instability near pinchoff that was seen in previous calculations 

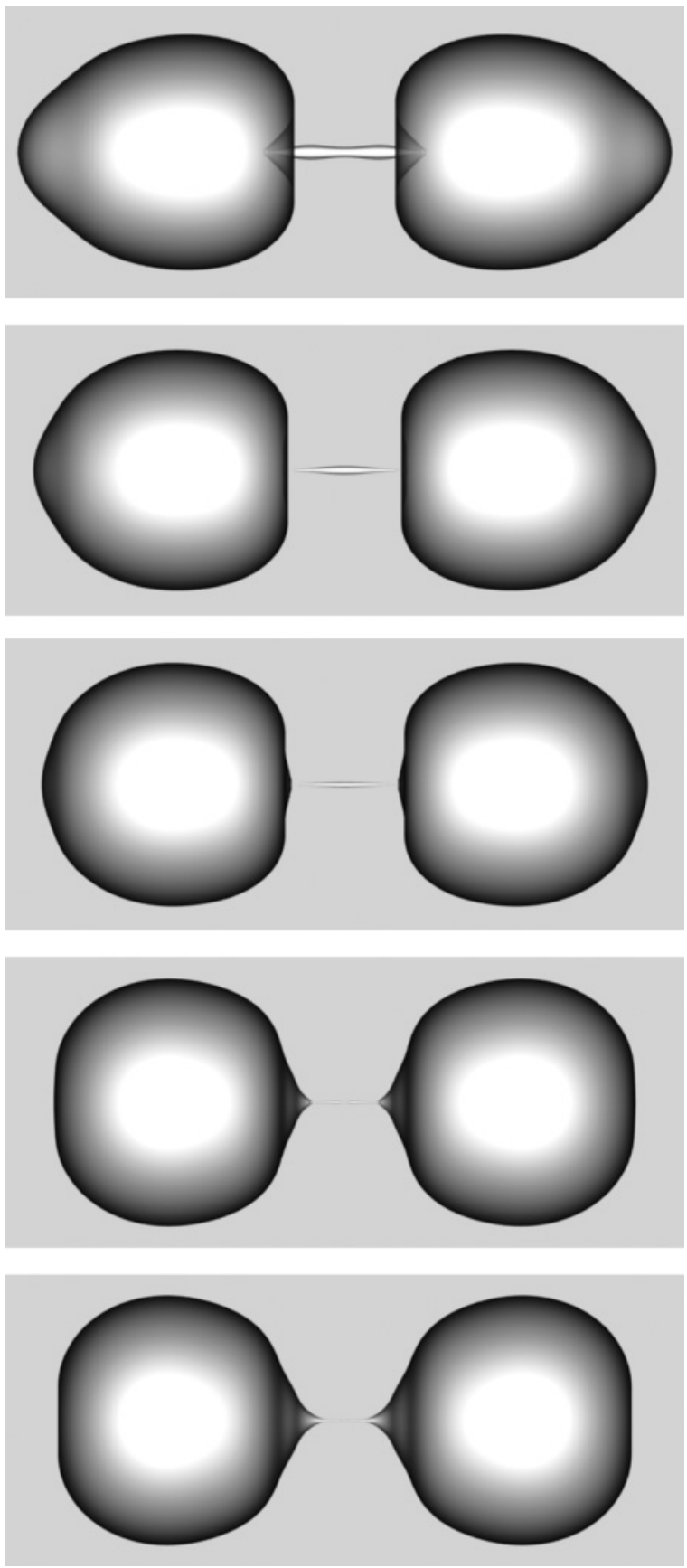

FIG. 27. 3D profiles at pinchoff for $D=0,1,2,4,6$ (top to bottom), geometry 2 . For the horizontal axis $0.4 \leqslant z \leqslant 6.6$ and the vertical axis $-1.33 \leqslant r \leqslant 1.33$.

$[6,7]$ is not present, and the lack of instability is consistent with the Burton and Taborek experiments.

To show that these results are not due to the mesh resolution, the front profiles for $D=6$ were computed with the coarse and finer grids, as explained in Sec. V A, and at pinchoff time are depicted in Fig. 6. Note that the overall front profile is almost indistinguishable and the difference in the axial coordinate at the separation point is less than $2 \%$. No instabilities were found up to the finer mesh resolution.

As shown before, the calculations have been continued after pinchoff for all $D$ values. In the range $0 \leqslant D \leqslant 10$ a satellite is
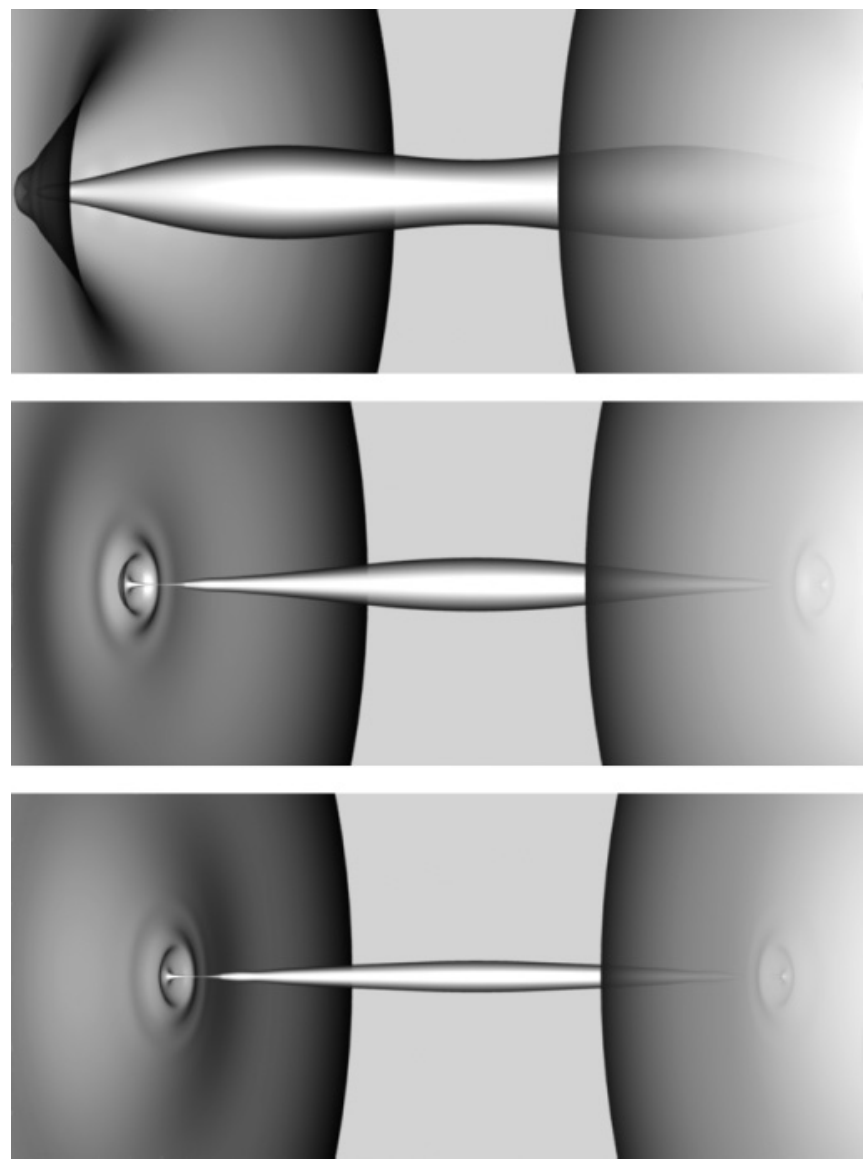

FIG. 28. 3D focused profiles at pinchoff for $D=0,1,2$ (top to bottom), $30^{\circ}$ view.
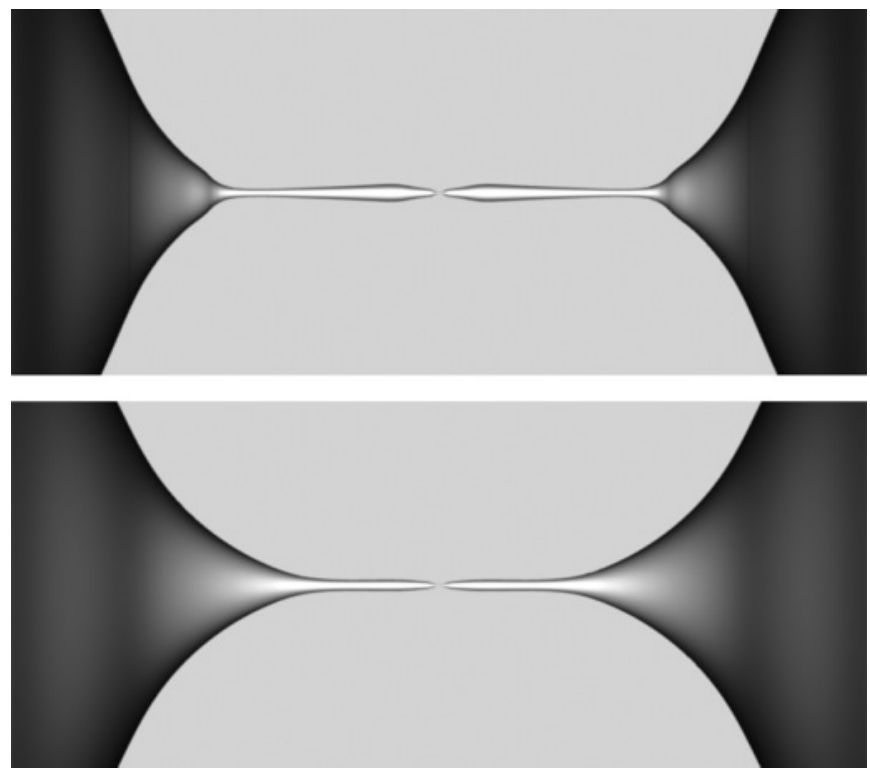

FIG. 29. 3D focused profiles at pinchoff for $D=4,6$ (top to bottom), front view. For the horizontal axis $2.9 \leqslant z \leqslant 4.1$ and the vertical axis $-0.26 \leqslant r \leqslant 0.26$. 

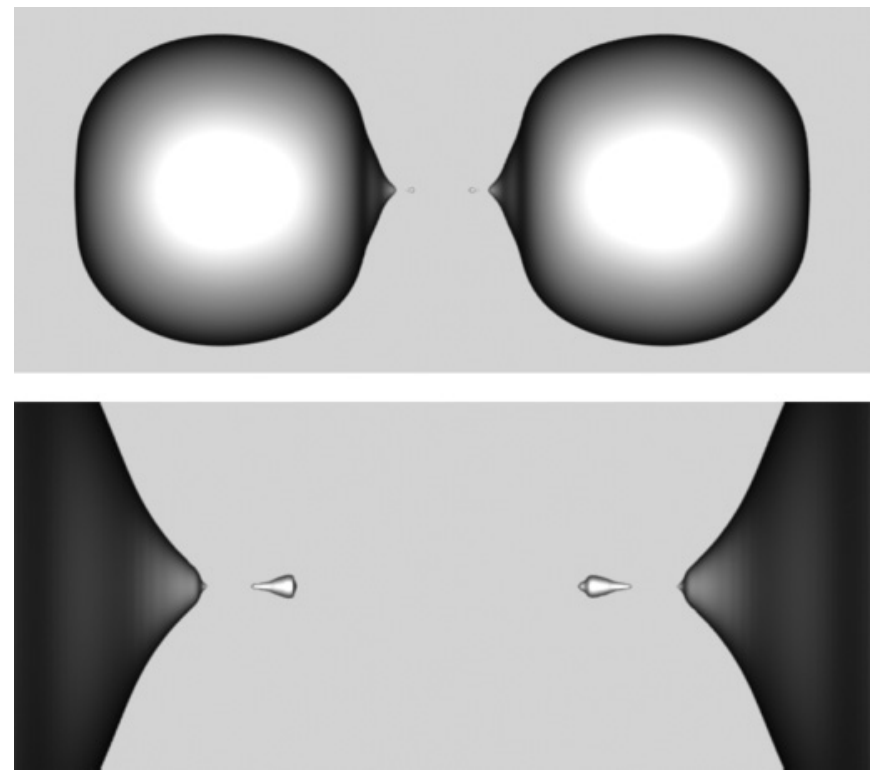

FIG. 30. 3D profiles after pinchoff for $D=4$, global and focused. For the horizontal axis $2.9 \leqslant z \leqslant 4.1$ and the vertical axis $-0.26 \leqslant$ $r \leqslant 0.26$.

formed and its volume decreases as $D$ increases. For $D=10$ a very tiny satellite is still present, whereas for $D=20$ and $D=100$ the flow is much faster and the satellite is absent.

The numbers for the scaling exponents, the satellite drop volume, and the front profiles at pinchoff would seem to indicate a continuous transition from droplet to bubble in the range $4<D<10$, rather than the sharp transition $D \approx 4$ predicted by Burton and Taborek, at least for drop geometry 1 . However, further analysis has shown that our results are in complete agreement with those of Burton and Taborek, when discrepancies in the initial drop geometry are taken into account. In Fig. 24, the computed scaling exponents for geometry 1 are compared with those reported in [5]. Experimental values in the range $0<D<1$ would be very useful for comparison, but they are not reported in [5].

To ascertain whether the transition range depends upon initial conditions, we ran a series of numerical experiments

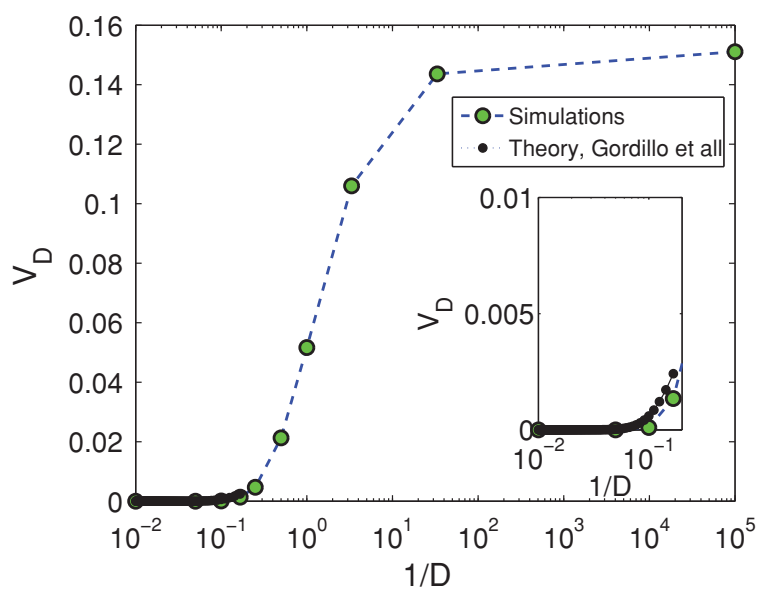

FIG. 31. (Color online) Satellite volume versus $D$, theoretical and calculated, geometry 1 . starting with a two lobe geometry drop, with an initial neck separating two equal inner fluid masses (geometry 2; see Fig. 3). The simulations were carried out for $D=$ $0,1,2,4,6$, with the same fixed domain $\Omega_{D}=[-2,2] \times[0,8]$ and finer mesh discretization parameters. For the free surface representation equally spaced nodes were chosen with fixed $\Delta s=0.03$, which led to $N_{p} \approx 250$ for all cases. For this set of numerical experiments, the conservation of the total system energy, calculated as in [11], was also monitored; the relative error at pinchoff time $e_{E}$ is shown in Table IV, together with $\alpha_{D}, V_{D}, t_{p}$, and $e_{V}$ obtained for each $D$ value. Inspecting Table IV and Fig. 25, where the computed and experimental scaling exponents are plotted for this drop geometry, we can conclude that the transition region is sharp and it occurs at $D \approx 4$, in perfect agreement with Burton and Taborek findings.

The slow decreasing behavior of the scaling exponent in the bubble regime has also been investigated for geometry 2 and $D=6$. Here we have used formula (24) to plot the theoretical $r_{\text {min }}=C_{1} \tau^{\alpha(\tau)}$ and the computed $r_{\text {min }}$ versus $\tau$. Figure 26 shows that there is a good agreement if we take $C_{1}=0.2$ in the range $0.005<r_{\min }<0.015$. The linear fittings to the computed behavior of $r_{\text {min }}$ lead to decreasing scaling exponents shown in Table V.

Finally we depict in Fig. 27 3D renderings of the drop profiles for $D=0,1,2,4,6$ at pinchoff time. For $D=0,1,2$ the drop profile overturns and we have focused the pinchoff region with a camera angle of $30^{\circ}$; the corresponding renderings are shown in Fig. 28. In the case of $D=4,6$ there is no overturning and focused profiles are shown in Fig. 29. The axial coordinate at pinchoff time is $z_{p}=3.5$ for $D=4,6$, and tiny satellites can be seen in Fig. 30 for $D=4$. For $D=6$ no satellite drop is developed up to the finer mesh resolution.

\section{Satellite volume}

Satellite size predictions are very useful in many technological applications. The calculated relative sizes of satellites corresponding to geometry 2 are smaller than those computed with geometry 1 when comparing the same $D$ values. In Fig. 31 we plot the volume of the satellite with respect to the density ratio $D$ for geometry 1 . It can be inferred that the formation mechanism of droplets and bubbles may differ significantly as the curve shows different behavior above and below the value $D=1$. For the bubble regime and geometry 1 we have compared the computed satellite volume with the theoretical formula (9) given in [19] and found good agreement for parameter values of $A=0.1$ and $\Gamma^{\star}=4$; see Fig. 31 .

\section{CONCLUSIONS}

By using the level set-boundary integral approach we have built up a seamless modeling and numerical methodology to study the evolution through pinchoff into post separation dynamics for a two inviscid fluid system. The calculations accurately predict the limiting Rayleigh case $D=0$ and bubble $D=\infty$ behaviors and no numerical instabilities are seen in the range $0 \leqslant D \leqslant 100$. Therefore, unlike previous computational work, it has been possible to compute scaling exponents for the minimum neck radius over the entire range of 
relative fluid density $D$. The transition range between droplet and bubble behavior has also been studied and it seems to be nonuniversal, as we have shown its dependency upon initial drop geometry. For an initial drop with a neck separating two equal inner fluid masses the transition zone is sharp and it happens at $D \approx 4$, in perfect agreement with experimental findings of Burton and Taborek. For a drop with nonequal fluid masses at both sides on the initial neck, the transition is milder and occurs in the range $4<D<10$.

\section{ACKNOWLEDGMENTS}

This work was supported by US Department of Energy, Applied Mathematical Sciences, the Division of Mathematical Sciences, National Science Foundation, and the Spanish Ministry of Science and Innovation (Project No. MTM201018427). The authors would like to thank J. Burton and M. A. Fontelos for their helpful discussions. The 3D renderings were done by F. M. Villalon with Blender.
[1] O. A. Basaran, AIChE J. 48, 1842 (2002).

[2] S. T. Thoroddsen, Nus. Eng. Res. News 22 (1), 10 (2007).

[3] J. Eggers, Rev. Mod. Phys. 69, 865 (1997).

[4] J. Eggers and E. Villermaux, Rep. Prog. Phys. 71, 036601 (2008)

[5] J. C. Burton and P. Taborek, Phys. Rev. Lett. 101, 214502 (2008).

[6] D. Leppinen and J. R. Lister, Phys. Fluids 15, 568 (2003).

[7] M. Nitsche and P. H. Steen, J. Comput. Phys. 200, 299 (2004).

[8] M. Garzon, L. J. Gray, and J. A. Sethian, J. Comput. Phys. 228, 6079 (2009).

[9] J. A. Sethian, Level Set Methods and Fast Marching Methods: Evolving Interfaces in Computational Geometry, Fluid Mechanics, Computer Vision, and Materials Science, Cambridge Monographs on Applied and Computational Mathematics (Cambridge University Press, Cambridge, 1999).

[10] M. Garzon, L. J. Gray, and J. A. Sethian, J. Comput. Appl. Math. (to be published).

[11] M. Garzon, L. J. Gray, and J. A. Sethian, Int. J. Numer. Meth Fluids (to be published).

[12] S. J. Osher and J. A. Sethian, J. Comput. Phys. 79, 12 (1988).
[13] D. Adalsteinsson and J. A. Sethian, J. Comput. Phys. 148, 2 (1999).

[14] M. Garzon, D. Adalsteinsson, L. J. Gray, and J. A. Sethian, Interf. and Free Bound. 7, 277 (2005).

[15] R. Malladi, J. A. Sethian, and B. C. Vemuri. IEEE Tans. on Pattern Anal. and Machine Intelligence 17(2), 158 (1995).

[16] L. J. Gray, M. Garzon, V. Mantič, and E. Graciani, Int. J. Numer. Meth. Engrg. 66, 2014 (2006).

[17] S. Gekle and J. M. Gordillo, J. Fluid Mech. 633, 293 (2010).

[18] See supplemental material at [http://link.aps.org/supplemental/ 10.1103/PhysRevE.83.046318] for movie of a drop evolution corresponding to $D=1$ case.

[19] J. M. Gordillo and M. A. Fontelos, Phys. Rev. Lett. 98, 144503 (2007).

[20] J. Eggers, M. A. Fontelos, D. Leppinen, and J. H. Snoeijer, Phys. Rev. Lett. 98, 094502 (2007).

[21] N. C. Keim, P. Moller, W. W. Zhang, and S. R. Nagel, Phys. Rev. Lett. 97, 144503 (2006).

[22] S. Gekle, J. H. Snoeijer, D. Lohse, and D. van der Meer, Phys. Rev. E 80, 036305 (2009). 